\title{
CRITICAL MARKOV BRANCHING PROCESS LIMIT THEOREMS ALLOWING INFINITE VARIANCE
}

\author{
ANTHONY G. PAKES, * University of Western Australia
}

\begin{abstract}
This paper gives easy proofs of conditional limit laws for the population size $Z_{t}$ of a critical Markov branching process whose offspring law is attracted to a stable law with index $1+\alpha$, where $0 \leq \alpha \leq 1$. Conditioning events subsume the usual ones, and more general initial laws are considered. The case $\alpha=0$ is related to extreme value theory for the Gumbel law.
\end{abstract}

Keywords: Markov branching process; limit theorem; extreme value theory; regular and rapid variation

2010 Mathematics Subject Classification: Primary 60J27; 60J80

Secondary 60F05; 60F 15

\section{Introduction}

Let $f(s)=\sum_{j \geq 0} p_{j} s^{j}$ denote the probability generating function (PGF) of the offspring law of the simple (Bienaymé-Galton-Watson) branching process. Assume that $p_{0}>0$ and that the mean per-capita number of offspring is $m=\sum j p_{j}=1$, i.e. the process is critical. Except for the last two sections, we also suppose that

$$
f(s)=s+(1-s)^{1+\alpha} L\left(\frac{1}{1-s}\right), \quad 0 \leq s<1,
$$

where $0 \leq \alpha \leq 1$ and $L(x)$ is slowly varying at infinity. This and related notions are described at the end of Section 2. Let $Z_{n}$ denote the population size of the $n$th generation, and let $\mathrm{P}_{i}(\cdot)=\mathrm{P}_{i}\left(\cdot \mid Z_{0}=i\right)$, and similarly for the expectation.

Slack (1968) proved that if $0<\alpha \leq 1$ then there are norming constants $Q_{n} \rightarrow 0$ such that $\mathrm{P}_{1}\left(Q_{n} Z_{n} \leq x \mid Z_{n}>0\right)$ converges weakly to a nondegenerate limit. An admissible choice of norming constant is $Q_{n}=\mathrm{P}_{1}\left(Z_{n}>0\right)$. Later, Slack (1972) proved that if such weak convergence holds with this particular norming, then (1.1) holds for some $\alpha \in(0,1]$ and slowly varying $L$.

Recently, Nagaev and Wachtel (2007) proved that if (1.1) holds with $\alpha=0$ then

$$
\mathrm{P}_{1}\left(L\left(Q_{n}^{-1}\right) V\left(Z_{n}\right) \leq z \mid Z_{n}>0\right) \rightarrow 1-\mathrm{e}^{-z}, \quad z>0
$$

where

$$
V(x)=\int_{0}^{1-1 / x} \frac{\mathrm{d} s}{f(s)-s}, \quad x \geq 1 .
$$

Received 6 July 2009; revision received 18 January 2010.

* Postal address: School of Mathematics and Statistics, University of Western Australia, 35 Stirling Highway, Crawley, WA 6009, Australia. Email address: pakes@maths.uwa.edu.au 
Let $\left(Z_{t}: t \geq 0\right)$ denote the Markov branching process (MBP) with split rate $\rho>0$ and offspring law $\left\{p_{j}\right\}$ as above, but with the conventionally added condition $p_{1}=0$. The MBP analogue of the above results can be obtained by applying them to discrete skeletons of the MBP, in the manner of Conner (1967). However, the aim of this paper is to exploit the smooth structure of the MBP to give simple direct proofs of more general conditional limit theorems. See Section 8 for remarks about what is meant by 'smooth structure'.

The case $\alpha>0$ is treated in Section 3 where three conditioning regimes are considered. Let $T=\inf \left\{t: Z_{t}=0\right\}$ denote the extinction time of the MBP, and let $\tau \geq 0$ be such that $\tau / t \rightarrow c \in[0, \infty]$ as $t \rightarrow \infty$. The three conditioning regimes are:

(a) $T>t+\tau$

(b) $t<T \leq t+\tau$; and

(c) $T>t+\tau$, where $\tau \rightarrow \infty$ and then $t \rightarrow \infty$.

The fundamental conditioning $T>t$ can be interpreted as $t<T \leq t+\tau$ with the limits taken as in (c). The fundamental conditioning was treated in Zolotarev (1957) using a direct manipulation of the integrated backward Kolmogorov equation. We shall reprise his proof in Section 3 in a more direct manner (Theorem 3.1). Results for regimes (a) and (b) then follow easily, excepting some boundary cases which need additional consideration. Regime (c) is simply the MBP version of what, in the context of Galton-Watson processes, is called in Athreya and Ney (1972, pp. 56-60) the $Q$-process. It is also the conditioning defining the 'doubly limiting conditional' theorem from the quasistationary theory of Markov processes (see Anderson (1991, p. 181)).

Representations of the limit laws are investigated in Section 4. These include integral representations for their densities (with respect to the Lebesgue measure), and some representations in terms of algebraic combinations of independent random variables.

If $R(y)$ denotes the inverse of the function $V(x)$ in (1.2) then $G(y)=1-1 / R(y)$ is a distribution function with support $[0, \infty)$, and differentiable in $(0, \infty)$. In the case that (1.1) holds with $\alpha>0$, we will see that $\bar{G}(y)=1-G(y)$ is regularly varying with index $-1 / \alpha$, i.e. it lies in the maximal domain of attraction of a Fréchet law. This observation is interesting, but not crucial for the proofs in Section 3. However, if $\alpha=0$ then $G$ is a von Mises distribution function, and, hence, attracted to the Gumbel law. This fact is crucial for our treatment in Section 5 of the fundamental conditioning in the case $\alpha=0$ (Theorem 5.1). Since $\mathrm{P}(T \leq t)=G(\rho t)$, it follows that the cases $\alpha>0$ and $\alpha=0$ correspond to the extinction time law being attracted to the Fréchet or the Gumbel law, respectively. We expand on these connections in Section 8. Alterations to our proof yields results for regimes (a) and (b) under a quite specific prescription for the dependence of $\tau$ on $t$. A kind of inversion of our approach for the fundamental conditioning yields a limit theorem for regime (c), but with norming which is qualitatively different to that for the fundamental conditioning.

In Sections 3 and 5, we examine the effect of different initial laws for the MBP. In Section 6 we give converse results showing that (1.1) is a consequence of a basic limit assertion. Finally, in Section 7 we obtain a conditional limit theorem which requires no moment assumption beyond finitude of $m$. This result is analogous to that obtained in Pakes (1999, Theorem 2.5) for the Galton-Watson process, which in turn stemmed from Seneta (1967).

In Section 2 we collect some representation formulae which will be used throughout, and also some notation and basic facts about regular and rapid variation. 


\section{Preliminary facts}

Recall that $\rho$ is the splitting rate of the MBP, i.e. individual lifetimes are independent with an exponential law having mean $\rho^{-1}$. Individual members of the population reproduce at the end of their lives according to the offspring law $\left\{p_{j}\right\}$. We follow the convention that $p_{1}=0$, so that splits correspond to observable changes in the population size. This, together with the assumption that $m=1$, ensures that the offspring law is nondegenerate. In particular, $0<p_{0}<1$. Defining $p_{i j}(t)=\mathrm{P}_{i}\left(Z_{t}=j\right)$ and $F(s, t)=\sum_{j \geq 0} p_{1 j}(t) s^{j}$, it follows from the branching property that

$$
\sum_{j \geq 0} p_{i j}(t) s^{j}=(F(s, t))^{i}, \quad i=1,2, \ldots
$$

The backward Kolmogorov equation can be expressed as

$$
\int_{s}^{F(s, t)} \frac{\mathrm{d} u}{f(u)-u}=\rho t .
$$

The MBP has an invariant measure $\left\{\mu_{j} ; j=1,2, \ldots\right\}$, i.e. $\sum_{i \geq 1} \mu_{i} p_{i j}(t)=\mu_{j}$ for $t \geq 0$ and $j \geq 1$, and it is unique up to constant factors (see Harris (1963, p. 110)). Adopting the normalization $\mu_{1}=1 / p_{0}$, the generating function of the invariant measure is

$$
\mathcal{M}(s)=\int_{0}^{s} \frac{\mathrm{d} u}{f(u)-u} .
$$

It follows that (2.1) takes the form $\mathcal{M}(F(s, t))=\rho t+\mathcal{M}(s)$. But (1.2) implies that $V(x)=$ $\mathcal{M}(1-1 / x)$, and recalling that $R(y)$ is the inverse of $V(x)$, we obtain the following basic representations:

$$
\frac{1}{1-F(s, t)}=R\left(\rho t+V\left(\frac{1}{1-s}\right)\right)
$$

and, defining $F(t)=\mathrm{P}_{1}(T \leq t)=F(0, t)$,

$$
\frac{1}{1-F(t)}=R(\rho t)
$$

Lemma 2.1. Assuming only that $m=1$, the function $V$ is concave increasing to $\infty$ and $R$ is convex increasing to $\infty$.

Proof. It follows from (1.2), with $s=1-x^{-1}$, that $V^{\prime}(x)=(1-s)^{2} /(f(s)-s)$. The right-hand side can be written as $(1-s) /(1-g(s))$, where $g(s)=(1-f(s)) /(1-s)$ is a PGF. The convexity of $g$ implies that, as a function of $s, V^{\prime}(x)$ decreases to $1 / g^{\prime}(1)$ as $s \uparrow 1$. Since $\mathrm{d} s / \mathrm{d} x=x^{-2}>0$, it follows that $V^{\prime \prime}(x)<0$.

Eliminating the time derivative term in the generating function forms of the backward and forward Kolmogorov equations (see Athreya and Ney (1972, p. 106)) gives the following useful identity:

$$
\frac{\partial}{\partial s} F(s, t)=\frac{a(F(s, t))}{a(s)},
$$

where $a(s)=\rho(f(s)-s)$.

Results about regular and slow variation are frequently used, and the salient facts are collected here. We refer the reader to Bingham et al. (1987) for more information. The class of functions 
which are slowly varying at $\infty$ is denoted by $\&$. Thus, $L \in \delta$ if it is defined on $(0, \infty)$ and, for each $\lambda>0, \lim _{x \rightarrow \infty} L(\lambda x) / L(x)=1$. The uniform convergence theorem asserts that this convergence holds uniformly with respect to $\lambda$ in compact subsets of $(0, \infty)$. There is a representation theorem asserting that if $L \in \delta$ then $L(x)=c(x) \exp \left(\int_{1}^{x}(\varepsilon(x) / x) \mathrm{d} x\right)$, where $c(x) \rightarrow c>0$ and $\varepsilon(x) \rightarrow 0$ as $x \rightarrow \infty$. Call $\varepsilon(x)$ the index function of $L$. If $c(x) \equiv c$ then $L$ is said to be normalized slowly varying, and $\varsigma_{0}$ denotes the set of normalized members of $\&$. A function $R(x)$ defined on $(0, \infty)$ is called regularly varying (at $\infty$ ) with index $\delta$ if $\lim _{x \rightarrow \infty} R(\lambda x) / R(x)=\lambda^{\delta}$ for all $\lambda>0$. Such a function can be expressed as $R(x)=x^{\delta} L(x)$ for some $L \in$ \&. A theorem of Lamperti (see Bingham et al. (1987, p. 59)) implies that if the derivative $R^{\prime}(x)$ exists and it is ultimately monotone, then $x R^{\prime}(x) / R(x)=\delta+o(1)$ as $x \rightarrow \infty$. Integrating this relation shows that the slowly varying factor $L \in \varsigma_{0}$.

Finally, we say that $R$ is rapidly varying (see Bingham et al. (1987, p. 83)) if

$$
\lim _{x \rightarrow \infty} \frac{R(\lambda x)}{R(x)}= \begin{cases}0 & \text { if } 0<\lambda<1 \\ \infty & \text { if } \lambda>1\end{cases}
$$

Assuming that (1.1) holds, (1.2) becomes

$$
V(x)=\int_{1}^{x} \frac{v^{\alpha-1} \mathrm{~d} v}{L(v)}, \quad x \geq 1 .
$$

It follows from Karamata's theorem (see Bingham et al. $\left(1987\right.$, p. 26)) that $V \in \mathcal{R}_{\alpha}$, and it follows from Lamperti's theorem that the slowly varying factor of $V$ is normalized.

We shall often use the convention that if a quantity $q \in[0,1]$, then $\bar{q}=1-q$. Thus, $\bar{F}(s, t)=1-F(s, t)$ and $\bar{F}(t)=1-F(t)$.

\section{The case $0<\alpha \leq 1$}

We begin by stating and proving the fundamental conditional limit theorem, i.e. the essence of Theorem 7 of Zolotarev (1957).

Theorem 3.1. If (1.1) with $0<\alpha \leq 1$ holds then

$$
\lim _{t \rightarrow \infty} \mathrm{P}_{1}\left(\bar{F}(t) Z_{t} \leq x \mid T>t\right)=D_{\alpha}(x),
$$

where

$$
\psi_{\alpha}(\theta)=\int_{0}^{\infty} \mathrm{e}^{-\theta x} \mathrm{~d} D_{\alpha}(x)=1-\left(1+\theta^{-\alpha}\right)^{-1 / \alpha} .
$$

Proof. Since $R$ increases (convexly) to $\infty$, it follows that $\bar{F}(t) \rightarrow 0$. We will let $s=s_{t}=$ $\mathrm{e}^{-\theta \bar{F}(t)}$ in (2.3). So $1-s_{t} \sim \theta \bar{F}(t)$, and it follows from the uniform convergence theorem and (2.4) that

$$
V\left(\frac{1}{1-s_{t}}\right) \sim \theta^{-\alpha} V\left(\frac{1}{\bar{F}(t)}\right)=\theta^{-\alpha} V(R(\rho t))=\rho t \theta^{-\alpha} .
$$

Since $V \in \mathcal{R}_{\alpha}$, its inverse $R \in \mathcal{R}_{1 / \alpha}$ (see Bingham et al. (1987, p. 29)), so, from (2.3) and the uniform convergence theorem,

$$
\frac{1}{\bar{F}\left(s_{t}, t\right)} \sim R\left(\left(1+\theta^{-\alpha}\right) \rho t\right) \sim\left(1+\theta^{-\alpha}\right)^{-1 / \alpha} R(\rho t) .
$$


It follows from (2.4) that

$$
\lim _{t \rightarrow \infty} \frac{\bar{F}\left(s_{t}, t\right)}{\bar{F}(t)}=\left(1+\theta^{-\alpha}\right)^{-1 / \alpha},
$$

i.e.

$$
\mathrm{E}_{1}\left(\mathrm{e}^{-\theta \bar{F}(t) Z_{t}} \mid T>t\right)=1-\frac{\bar{F}(s, t)}{\bar{F}(t)} \rightarrow \psi_{\alpha}(\theta) .
$$

The assertion follows from the continuity theorem for Laplace-Stieltjes transforms, and $D_{\alpha}(x)$ is nondefective because $\psi(0+)=1$.

Remark 3.1. It follows from the proof that $\bar{F} \in \mathcal{R}_{-1 / \alpha}$ with $\alpha>0$ is equivalent to (1.1).

Zolotarev (1957) began the proof of his Theorem 7 using the integrated backward equation essentially as above, but he reached (3.2) in a somewhat indirect way using a proof by contradiction. Expression (3.1) for $\psi_{\alpha}(\theta)$ is a minor algebraic rearrangement of the form he obtained. However, his expression for $D_{\alpha}$ is not correct, though comparing it with the expression near the top of page 250 suggests that his error is an inadvertent omission of an algebraic factor. We derive his (corrected) integral representation in the next section.

Suppose now that $Z_{0}$ has a law whose PGF is

$$
\pi(s)=1-(1-s)^{\delta} \mathcal{L}\left(\frac{1}{1-s}\right) \text { and } \pi(0)=0,
$$

where $0 \leq \delta \leq 1$ and $\mathcal{L} \in \&$. The case $\delta=1$ includes any initial law having a finite first moment. It follows from (3.2) and the uniform convergence theorem applied to $1-\pi(1-1 / x) \in$ $\mathcal{R}_{-\delta}$ that

$$
\lim _{t \rightarrow \infty} \frac{1-\pi\left(F\left(s_{t}, t\right)\right)}{1-\pi(F(t))}=\left(1+\theta^{-\alpha}\right)^{-\delta / \alpha} .
$$

Let $\mathrm{E}_{\pi}(\cdot)$ and $\mathrm{P}_{\pi}(\cdot)$ denote the conditional expectation and probability given the initial law $\pi$. Since

$$
\mathrm{E}_{\pi}\left(s^{Z_{t}} \mid T>t\right)=\frac{\pi(F(s, t))-\pi(F(t))}{1-\pi(F(t))},
$$

the next result follows from (3.4).

Theorem 3.2. Suppose that (1.1) holds with $0<\alpha \leq 1$ and that (3.3) holds. If $0<\delta \leq 1$ then

$$
\lim _{t \rightarrow \infty} \mathrm{P}_{\pi}\left(\bar{F}(t) Z_{t} \leq x \mid T>t\right)=D_{\alpha, \delta}(x)
$$

where

$$
\psi_{\alpha, \delta}(\theta)=\int_{0}^{\infty} \mathrm{e}^{-\theta x} \mathrm{~d} D_{\alpha, \delta}(x)=1-\left(1+\theta^{-\alpha}\right)^{-\delta / \alpha} .
$$

If $\delta=0$ then, given that $T>t, \bar{F}(t) Z_{t} \stackrel{\mathrm{P}}{\rightarrow} \infty$.

Remark 3.2. Since $D_{\alpha, 1}(x)=D_{\alpha}(x)$, the case $\alpha=1$ of Theorem 3.2 is a real generalization of Theorem 3.1.

The next results deal with the conditioning regimes (a) and (b), for which we need the following lemma. 
Lemma 3.1. Suppose that the conditions of Theorem 3.1 hold, and that $\tau>0$ is a function of $t$ satisfying $\lim _{t \rightarrow \infty} \tau / t=c \in[0, \infty]$. Then

$$
\begin{gathered}
\lim _{t \rightarrow \infty} \frac{\bar{F}(t+\tau)}{\bar{F}(t)}=(1+c)^{-1 / \alpha}, \\
\lim _{t \rightarrow \infty} \frac{\bar{F}(\tau)}{\bar{F}(t)}=c^{-1 / \alpha} \leq \infty,
\end{gathered}
$$

and, recalling that $s_{t}=\exp (-\theta \bar{F}(t))$,

$$
\lim _{t \rightarrow \infty} \frac{\bar{F}\left(s_{t} F(\tau), t\right)}{\bar{F}(t)}=\left[1+\left(\theta+c^{-1 / \alpha}\right)^{-\alpha}\right]^{-1 / \alpha} \leq 1 .
$$

Proof. If $0<c<\infty$ then limits (3.6) and (3.7) follow from Remark 3.1 and the uniform convergence theorem. For the same reasons, (3.6) is valid if $c=0$. Both are valid if $c=\infty$ because, since $\bar{F}$ is decreasing, the uniform convergence theorem extends to uniformity in any semi-infinite interval $\left[\lambda^{\prime}, \infty\right)$, where $\lambda^{\prime}>0$. See Bingham et al. (1987, p. 22). If $c=0$ then, given $\epsilon>0$, there exists $t^{\prime}$ such that $\tau / t \leq \epsilon$ if $t \geq t^{\prime}$. Hence,

$$
\liminf _{t \rightarrow \infty} \frac{\bar{F}(\tau)}{\bar{F}(t)} \geq \lim _{t \rightarrow \infty} \frac{\bar{F}(\epsilon t)}{\bar{F}(t)}=\epsilon^{-1 / \alpha} .
$$

Letting $\epsilon \rightarrow 0$ gives (3.7). Thus, the values of limits (3.6) and (3.7) are 1 and $\infty$, respectively, if $c=0$, and both are 0 if $c=\infty$.

For (3.8), observe that

$$
1-s_{t} F(\tau)=1-s_{t}+\bar{F}(\tau)-\left(1-s_{t}\right) \bar{F}(\tau)=\theta \bar{F}(t)(1+o(1))+\bar{F}(\tau)+O(\bar{F}(t) \bar{F}(\tau)) .
$$

If $0<c \leq \infty$ then the right-hand side is asymptotically equal to $\left(\theta+c^{-1 / \alpha}\right) \bar{F}(t)$ and (3.8) follows from (3.2) and its local uniform convergence with respect to $\theta$.

If $c=0$ then (3.7) implies that $\left(1-s_{t} F(\tau)\right) / \bar{F}(t) \rightarrow \infty$. Hence, given a (large) positive constant $M$, there exists $t^{\prime}>0$ such that $s_{t} F(\tau)<1-M \bar{F}(t) \in(0,1)$ if $t>t^{\prime}$. So

$$
1>\frac{\bar{F}(t+\tau)}{\bar{F}(t)}>\frac{\bar{F}\left(s_{t} F(\tau), t\right)}{\bar{F}(t)}>\frac{\bar{F}(1-M \bar{F}(t), t)}{\bar{F}(t)} \rightarrow \bar{\psi}_{\alpha}(M) .
$$

The right-hand side converges to unity as $M \rightarrow \infty$, and (3.8) follows.

Since $\mathrm{P}_{\pi}\left(Z_{t}=j, T>t+\tau\right)=\mathrm{P}_{\pi}\left(Z_{t}=j\right)\left(1-F^{j}(\tau)\right)$, it follows that

$$
\mathrm{E}_{\pi}\left(s^{Z_{t}} \mid T>t+\tau\right)=\frac{\pi(F(s, t))-\pi(F(s F(\tau), t))}{1-\pi(F(t+\tau))} .
$$

It follows from (3.2), (3.6), (3.8), and (3.3) that if $0 \leq c<\infty$ then

$$
\widehat{A}_{\alpha, c, \delta}(\theta):=\lim _{t \rightarrow \infty} \mathrm{E}_{\pi}\left(s_{t}^{Z_{t}} \mid T>t+\tau\right)=\frac{\left[1+\left(\theta+c^{-1 / \alpha}\right)^{-\alpha}\right]^{-\delta / \alpha}-\left[1+\theta^{-\alpha}\right]^{-\delta / \alpha}}{(1+c)^{-\delta / \alpha}} .
$$

Here and below, we use the convention that $\widehat{A}(\theta)$ denotes the Laplace-Stieltjes transform of a distribution function $A(x)$. If $c=\infty$, it follows from (3.6) that these limit operations result in an indeterminate form. Theorem 3.4, below, addresses this case. Observing that, for $c<\infty$,

$$
\widehat{A}_{\alpha, c, \delta}(\theta)=\frac{\psi_{\alpha, \delta}(\theta)-\psi_{\alpha, \delta}\left(\theta+c^{-\alpha}\right)}{\bar{\psi}_{\alpha, \delta}\left(c^{-1 / \alpha}\right)},
$$

we have the following result for regime (a). 
Theorem 3.3. Suppose that the conditions of Theorem 3.2 hold with $0<\delta \leq 1$, and that $\tau>0$ is a function of $t$ satisfying $\lim _{t \rightarrow \infty} \tau / t=c \in[0, \infty)$. Then

$$
\lim _{t \rightarrow \infty} \mathrm{P}_{\pi}\left(\bar{F}(t) Z_{t} \leq x \mid T>t+\tau\right)=A_{\alpha, c, \delta}(x)=\frac{\int_{0}^{x}\left(1-\mathrm{e}^{-v c^{-1 / \alpha}}\right) \mathrm{d} D_{\alpha, \delta}(v)}{\int_{0}^{\infty}\left(1-\mathrm{e}^{-v c^{-1 / \alpha}}\right) \mathrm{d} D_{\alpha, \delta}(v)} .
$$

It follows from (3.11), and the evaluation of (3.8) as unity if $c=0$, that

$$
\lim _{c \rightarrow 0} A_{\alpha, c, \delta}(x) \rightarrow A_{\alpha, 0, \delta}(x)=D_{\alpha, \delta}(x) .
$$

We begin discussion of the case $c=\infty$ by noting that a binomial expansion yields the following corollary.

Corollary 3.1. We have

$$
\widehat{A}_{\alpha, \infty, \delta}(\theta):=\lim _{c \rightarrow \infty} \widehat{A}_{\alpha, c, \delta}(\theta)= \begin{cases}\bar{\psi}_{\alpha}^{\prime}(\theta)=\left(1+\theta^{\alpha}\right)^{-1-1 / \alpha} & \text { if } \delta=1, \\ 0 & \text { if } \delta<1 .\end{cases}
$$

The following preliminary result for the case $c=\infty$ starts from (3.9) and does not require the regular variation assumptions in (1.1) and (3.3). Recall that $a(s)=\rho(f(s)-s)$.

Lemma 3.2. If $m=1$ but $f(s)$ is otherwise arbitrary, then

$$
\mathrm{E}_{1}\left(s^{Z_{t}} \mid Z_{\infty}>0\right):=\lim _{\tau \rightarrow \infty} \mathrm{E}_{1}\left(s^{Z_{t}} \mid T>t+\tau\right)=s \frac{a(F(s, t))}{a(s)} .
$$

If, in addition, $\pi$ is an arbitrary initial law supported on the positive integers and with first moment $m_{\pi}$, then

$$
\lim _{\tau \rightarrow \infty} \mathrm{E}_{\pi}\left(s^{Z_{t}} \mid T>t+\tau\right)= \begin{cases}\mathrm{E}_{1}\left(s^{Z_{t}} \mid Z_{\infty}>0\right) \frac{\pi^{\prime}(F(s, t))}{m_{\pi}} & \text { if } m_{\pi}<\infty \\ 0 & \text { if } m_{\pi}=\infty .\end{cases}
$$

Proof. Let $\pi(s)=s$ in (3.9), and observe that, by virtue of the mean value theorem and $F(\tau) \rightarrow 1$, the numerator is asymptotically proportional to

$$
s \bar{F}(\tau) \frac{\partial}{\partial s} F(s, t)=s \bar{F}(\tau) \frac{a(F(s, t))}{a(s)}, \quad 0 \leq s<1,
$$

where the equality follows from (2.5). It follows from $\mathrm{E}_{1}\left(Z_{t}\right)=1$ for all $t$ that

$$
\bar{F}(t+\tau)=\bar{F}(F(\tau), t) \sim \bar{F}(\tau), \quad \tau \rightarrow \infty,
$$

and (3.12) follows from these estimates. In addition, (2.5) implies that the right-hand side of (3.12) tends to unity as $s \rightarrow 1$, i.e. the discrete limit law inherent in (3.12) is honest.

For general $\pi$, the mean value theorem yields

$$
\mathrm{E}_{\pi}\left(s^{Z_{t}} \mid T>t+\tau\right)=\mathrm{E}_{1}\left(s^{Z_{t}} \mid T>t+\tau\right) \frac{\pi^{\prime}\left(\zeta_{1}(\tau)\right)}{\pi^{\prime}\left(\zeta_{2}(\tau)\right)},
$$

where

$$
F(s F(\tau), t)<\zeta_{1}(\tau)<F(s, t) \text { and } F(t+\tau)<\zeta_{2}(\tau)<1 .
$$

So $\zeta_{1}(\tau) \rightarrow F(s, t)$ and $\zeta_{2}(\tau) \rightarrow 1$ as $\tau \rightarrow \infty$, and the second assertion follows from (3.12). 
The quotient on the right-hand side of (3.12) can be expressed as

$$
\exp \left(-\rho \int_{0}^{t}\left(1-f^{\prime}(F(s, v)) \mathrm{d} v\right)\right)
$$

and this is the PGF of an MBP subject to an external source of immigration. Immigration occurs as a compound Poisson process whose jump rate is $\rho$, and whose jump law has the PGF $f^{\prime}(s)$. Athreya and Ney (1972) used the term $Q$-process in the corresponding discrete-time context. The factor $\pi^{\prime}(F(s, t)) / m_{\pi}$ is the generating function of an independent MBP whose initial law has the PGF $\pi^{\prime}(s) / m_{\pi}$. It follows from Lemma 3.2 that regime (c) can yield a meaningful limit theorem only if $m_{\pi}<\infty$. In this case, any limit operation involving $F\left(s_{t}, t\right) \rightarrow 1$ will give a limit which is independent of $\pi$. So it suffices to consider only (3.12).

The next result covers regime (a) with $c=\infty$ and regime (c). Let $F^{\prime}(s, t)$ denote the derivative on the left-hand side of (2.5).

Theorem 3.4. If (1.1) holds with $0<\alpha \leq 1$ and $m_{\pi}<\infty$,

(i) then

$$
\lim _{t \rightarrow \infty} \mathrm{P}_{\pi}\left(\bar{F}(t) Z_{t} \leq x \mid Z_{\infty}>0\right)=A_{\alpha, \infty}(x)
$$

where

$$
\widehat{A}_{\alpha, \infty}(\theta)=\left(1+\theta^{\alpha}\right)^{-1-1 / \alpha} ;
$$

(ii) and if, in addition, $\tau / t \rightarrow \infty$, then

$$
\lim _{t \rightarrow \infty} \mathrm{P}_{\pi}\left(\bar{F}(t) Z_{t} \leq x \mid T>t+\tau\right)=A_{\alpha, \infty}(x) .
$$

Proof. We prove (ii) only, since (i) is very similar. Let $s=s_{t}=\mathrm{e}^{-\theta \bar{F}(t)}$ in (3.9). The arguments of $\pi$ there all tend to unity, and since $m_{\pi}$ is finite, it follows from the mean value theorem that the limit of the right-hand side is the same as for the case where $\pi(s)=s$. Assuming this form, the numerator is asymptotically equal to $\bar{F}(\tau) F^{\prime}\left(\zeta_{t}, t\right)$, where $s_{t} F(\tau)<$ $\zeta_{t}<s_{t}$. It follows from (3.7) with $c=\infty$ that $1-s_{t} \sim \theta \bar{F}(t)$, implying that $1-\zeta_{t} \sim \theta \bar{F}(t)$. So (3.2) and (1.1) yield

$$
F^{\prime}\left(\zeta_{t}, t\right)=\frac{a\left(F\left(\zeta_{t}, t\right)\right)}{a\left(\zeta_{t}\right)}=\frac{\left(\bar{F}\left(\zeta_{t}, t\right)\right)^{1+\alpha} L\left(1 / \bar{F}\left(\zeta_{t}, t\right)\right)}{(\theta \bar{F}(t))^{1+\alpha} L(1 / \bar{F}(t))} \rightarrow\left(1+\theta^{\alpha}\right)^{-1-1 / \alpha} .
$$

Finally, (3.6) and (3.7) imply that $\bar{F}(\tau) / \bar{F}(t+\tau) \rightarrow 1$, so we conclude that

$$
\mathrm{E}_{1}\left(\mathrm{e}^{-\bar{\theta} F(t) Z_{t}} \mid T>t+\tau\right) \rightarrow \widehat{A}_{\alpha, \infty}(\theta),
$$

and the theorem follows since $\widehat{A}_{\alpha, \infty}(0+)=1$.

Let $A_{\alpha, c}(x):=A_{\alpha, c, 1}(x)$, and similarly for the corresponding Laplace-Stieltjes transforms. It follows from Theorems 3.3 and 3.4, and Corollary 3.1, that $\left\{A_{\alpha, c}: 0 \leq c \leq \infty\right\}$ comprises a family of nondegenerate distribution functions. This remark is related to a functional limit theorem which is essentially a corollary of Theorem 3.1. Following Durrett (1978), with some altered notation, let $0<\zeta \leq 1$ and define the 'conditioned' process

$$
V_{t}^{+}(\zeta)=\left(\bar{F}(t) Z_{\zeta t} \mid Z_{t}>0, Z_{0}=i\right)
$$


Durrett's setting is the weak convergence of a sequence of continuous-time processes obtained by linear interpolation of discrete-time processes. His general setting and assumptions can be adapted to our setting with the result that $\left(V_{t}^{+}(\zeta): 0<\zeta \leq 1\right)$ converges weakly in the space $D[0,1]$ to an inhomogeneous Markov process $w(\zeta)$. The one-dimensional law of this limit process has the Laplace-Stieltjes transform $\eta_{\alpha}(\theta, \zeta)=\widehat{A}_{\alpha, c}\left(\zeta^{1 / \alpha} \theta\right)$, where $c=\zeta^{-1}-1$. The limit $\zeta \rightarrow 0+$ corresponds to $c \rightarrow \infty$, but the norming factor $\zeta^{1 / \alpha}$ has the effect of ensuring that $\eta_{\alpha}(\theta, 0+)=1$, i.e. $w(\zeta) \stackrel{\mathrm{P}}{\rightarrow} 0$, and, hence, that $\{0\}$ is an unattainable entrance state for the limit process. In addition, it follows from (3.10) that $\zeta^{-1 / \alpha} w(\zeta)$ converges in law (as $\zeta \rightarrow 0+$ ) to the same limit as in Corollary 3.1. Details of the application of Durett's theorem are given in Vatutin et al. (2008) for the Galton-Watson process satisfying (1.1).

Turning now to regime (b), it turns out, for reasons explained in the next section, that neither $\bar{F}(t)$ or $\bar{F}(\tau)$ are entirely satisfactory as norming functions. The following result uses a composite norming which is satisfactory.

Theorem 3.5. Suppose that (1.1) and (3.3) hold with $0<\alpha, \delta \leq 1$, and that $\tau>0$ is a function of t satisfying $\lim _{t \rightarrow \infty} \tau / t=c \in(0, \infty]$. Then the limit

$$
\lim _{t \rightarrow \infty} \mathrm{P}_{\pi}\left((\bar{F}(t)+\bar{F}(\tau)) Z_{t} \leq x \mid t<T \leq t+\tau\right)=B_{\alpha, c, \delta}(x)
$$

exists, where

$$
\widehat{B}_{\alpha, c, \delta}(\theta)=\frac{\psi_{\alpha, \delta}\left(\left(1+c^{-1 / \alpha}\right) \theta+c^{-1 / \alpha}\right)}{\psi_{\alpha, \delta}\left(c^{-1 / \alpha}\right)} .
$$

Proof. Similarly to (3.9),

$$
\mathrm{E}_{\pi}\left(s^{Z_{t}} \mid t<T \leq t+\tau\right)=\frac{\pi(F(s F(\tau), t))-\pi(F(t))}{\pi(F(t+\tau))-\pi(F(t))} .
$$

Choosing $s=s^{\prime}=\mathrm{e}^{-(\bar{F}(t)+\bar{F}(\tau)) \theta}$ we have, from (3.7),

$$
1-s^{\prime} F(\tau)=1-s^{\prime}+\bar{F}(\tau)(1+o(1)) \sim\left[\left(1+c^{-1 / \alpha}\right) \theta+c^{-1 / \alpha}\right] \bar{F}(t) .
$$

So (3.2) implies that

$$
\frac{\bar{F}\left(s^{\prime} F(\tau), t\right)}{\bar{F}(t)} \rightarrow \bar{\psi}_{\alpha}\left(\left(1+c^{-1 / \alpha}\right) \theta+c^{-1 / \alpha}\right) .
$$

Next, (3.6) implies that

$$
F(t+\tau)-F(t) \sim\left[1-(1+c)^{-1 / \alpha}\right] \bar{F}(t)=\psi_{\alpha}\left(c^{-1 / \alpha}\right) \bar{F}(t) .
$$

The assertion for $\delta=1$ follows from these estimates. The outcome for $\delta<1$ follows from the same estimates since, for example, using (3.3) and (3.6), the denominator in (3.13) is

$$
(\bar{F}(t))^{\delta} \mathcal{L}\left(\frac{1}{\bar{F}(t)}\right)-(\bar{F}(t+\tau))^{\delta} \mathcal{L}\left(\frac{1}{\bar{F}(t+\tau)}\right) \sim \psi_{\alpha, \delta}\left(c^{-1 / \alpha}\right)(\bar{F}(t))^{\delta} .
$$

The assertion follows from these estimates.

Note that if $c=\infty$ then the norming function is equivalent to $\bar{F}(t)$, and that $\widehat{B}_{\alpha, \infty, \delta}(\theta)=$ $\psi_{\alpha, \delta}(\theta)$. The first part of the following corollary is immediately evident from Theorem 3.5 , and the second part follows from the relation $1-\psi_{\alpha, \delta}\left(c^{-1 / \alpha}\right)=1-(1+c)^{-\delta / \alpha}$, implying that $\psi_{\alpha, \delta}(\theta) \sim \delta \theta^{-\alpha} / \alpha$ as $\theta \rightarrow \infty$. Here and below, the standard gamma law with (shape) parameter $\ell$ is defined by the density function $g_{\ell}(x)=x^{\ell-1} \mathrm{e}^{-x} / \Gamma(\ell)$, and $G_{\ell}(x)$ denotes its distribution function. 
Corollary 3.2. We have

$$
B_{\alpha, c, \delta}(x) \rightarrow \begin{cases}D_{\alpha, \delta}(x) & \text { as } c \rightarrow \infty \\ G_{\alpha}(x) & \text { as } c \rightarrow 0\end{cases}
$$

Note that the second limit is independent of $\delta$. The next result shows that this gamma law occurs as the limit law when $c=0$, even if $\delta=0$.

Theorem 3.6. Suppose that (1.1) holds with $0<\alpha \leq 1$, that (3.3) holds with $0 \leq \delta \leq 1$, and that $\tau \rightarrow \infty$ with $c=0$. Then

$$
\lim _{t \rightarrow \infty} \mathrm{P}_{\pi}\left(\bar{F}(\tau) Z_{t} \leq x \mid t<T \leq t+\tau\right)=G_{\alpha}(x) .
$$

Proof. Note that (3.7) with $c=0$ implies that the norming in the assertion effectively coincides with that used for Theorem 3.5. The condition $\tau \rightarrow \infty$ ensures that $\bar{F}(\tau) \rightarrow 0$, and, hence, that $1-s_{\tau} \sim \theta \bar{F}(\tau)$. It follows from (2.3) and (2.4) that

$$
\frac{1}{\bar{F}\left(s_{\tau} F(\tau), t\right)}-\frac{1}{\bar{F}(t)}=R\left(\rho t+V\left(\frac{1}{1-s_{\tau} F(\tau)}\right)\right)-R(\rho t) .
$$

Since $V \in \mathcal{R}_{\alpha}$,

$$
V\left(\frac{1}{1-s_{\tau} F(\tau)}\right) \sim(1+\theta)^{-\alpha} V\left(\frac{1}{\bar{F}(\tau)}\right)=(1+\theta)^{-\alpha} \rho \tau .
$$

Since $c=0$ and $R \in \mathcal{R}_{1 / \alpha}$, the mean value theorem and the uniform convergence theorem imply that the right-hand side of (3.14) is asymptotically proportional to $(1+\theta)^{-\alpha} \rho \tau R^{\prime}(\rho t)$. Lamperti's theorem implies that $R^{\prime}(\rho t) \sim R(\rho t) / \alpha \rho t$. Combining these estimates and rearranging (3.14) leads to the estimate

$$
F\left(s_{\tau} F(\tau), t\right)-F(t) \sim(1+\theta)^{-\alpha} \frac{\tau \bar{F}(t)}{\alpha t}, \quad t \rightarrow \infty .
$$

Next, $F(t+\tau)-F(t) \sim \tau F^{\prime}\left(\zeta_{t}\right)$, where, since $c=0, \zeta_{t} \sim t$. It follows from (2.3) that

$$
F^{\prime}(t)=\frac{\rho R^{\prime}(\rho t)}{(R(\rho t))^{2}}=\rho \bar{F}(t) \frac{R^{\prime}(\rho t)}{R(\rho t)} \sim \frac{\bar{F}(t)}{\alpha t},
$$

where Lamperti's theorem was used for the last step. In particular, $F^{\prime}(t)$ is regularly varying, and, hence, we obtain the estimate

$$
F(t+\tau)-F(t) \sim \frac{\tau \bar{F}(t)}{\alpha t} .
$$

Combining this with (3.15) yields

$$
\mathrm{E}_{1}\left(\mathrm{e}^{-\theta \bar{F}(\tau) Z_{t}} \mid t<T \leq t+\tau\right) \rightarrow(1+\theta)^{-\alpha},
$$

giving the assertion for the case $\pi(s)=s$.

It follows from the above estimates that if $F(t)<u_{t}<\max \left(F(t+\tau), F\left(s_{\tau} F(\tau), t\right)\right)$, then $1-u_{t} \sim \bar{F}(t)$. However,

$$
\mathrm{E}_{\pi}\left(s_{\tau}^{Z_{t}} \mid t<T \leq t+\tau\right)=\mathrm{E}_{1}\left(s_{\tau}^{Z_{t}} \mid t<T \leq t+\tau\right) \frac{\pi^{\prime}\left(u_{1 t}\right)}{\pi^{\prime}\left(u_{2 t}\right)},
$$

where $1-u_{i t} \sim \bar{F}(t), i=1,2$. It follows, from (3.3) and the uniform convergence theorem, 
that the quotient on the right-hand side tends to unity independently of the value of $\delta$. Thus, the assertion follows in general.

Conditional limit theorems for the time to extinction are easily obtained from the identity

$$
\mathrm{P}_{\pi}\left(\frac{T-t}{t} \leq z \mid T>t\right)=1-\frac{\bar{\pi}(F(t+t z))}{\bar{\pi}(F(t))},
$$

and expressing (3.6) as

$$
\lim _{t \rightarrow \infty} \frac{\bar{F}(t+t z)}{\bar{F}(t)}=(1+z)^{-1 / \alpha} .
$$

Theorem 3.7. If the assumptions of Theorem 3.2 hold with $0<\delta \leq 1$, and if $z \geq 0$, then

$$
\text { (i) } \lim _{t \rightarrow \infty} \mathrm{P}_{\pi}\left(\frac{T-t}{t} \leq z \mid T>t\right)=1-(1+z)^{-\delta / \alpha} \text {; }
$$

and

$$
\text { (ii) } \lim _{i \rightarrow \infty} \mathrm{P}_{i}\left(\frac{\rho T}{V(i)} \leq z\right)=\exp \left(-z^{-1 / \alpha}\right)
$$

\section{Representations of the limit laws}

We begin by defining the notation used below. Let $S_{\alpha}$ denote a random variable having the positive stable law with index $\alpha$, i.e. $\mathrm{E}\left(\exp \left(-\theta S_{\alpha}\right)\right)=\exp \left(-\theta^{\alpha}\right)$, and $\sigma_{\alpha}(x)$ denotes its density. Thus, $\mathrm{P}\left(S_{1}=1\right)=1$, and $\sigma_{1}$ is interpreted as a Dirac delta located at unity. Next, $\gamma(\ell)$ denotes a random variable having the standard gamma $(\ell)$ law. Denote by $\beta(a, b)$ a random variable which has a beta law with parameters $a, b>0$. This notation is extended so that $\beta(a, 1-a)$ is defined if $0<a \leq 1$ with the understanding that $\beta(1,0)=1$. The terms in sums and products of random variables will be understood to be independent. We write $X \stackrel{\mathrm{L}}{=} Y$ to denote equality in law of the random variables $X$ and $Y$.

On occasion we will use the known moment formula

$$
\mathrm{E}\left(S_{\alpha}^{-t}\right)=\frac{\Gamma(1+t / \alpha)}{\Gamma(1+t)}, \quad t>-\alpha .
$$

The simplest proof of this formula involves taking the expectation of the identity

$$
S_{\alpha}^{-t}=\frac{1}{\Gamma(t)} \int_{0}^{\infty} \mathrm{e}^{-\theta S_{\alpha}} \mathrm{d} \theta
$$

and integrating. Shanbhag and Sreehari (1977) derived this moment formula from their mixture representation $\left(\gamma(1) / S_{\alpha}\right)^{\alpha} \stackrel{\mathrm{L}}{=} \gamma(1)$.

Let $Y$ be a nonnegative random variable with distribution function $K$ not degenerate at 0 and with finite first moment $\mu_{K}$. The stationary-excess operation on $K$ is defined by

$$
\widetilde{K}(x)=\mu_{K}^{-1} \int_{0}^{x} \bar{K}(v) \mathrm{d} v,
$$

and we write $\tilde{Y}$ to denote a random variable whose distribution function is $\widetilde{K}$. Thus, $\widetilde{Y}$ represents times to the next renewal event in a stationary renewal process with generic interevent time $Y$. The length-biased version of $K$ is the distribution function $\mu_{K}^{-1} \int_{0}^{x} v \mathrm{~d} K(v)$, and $\widehat{Y}$ denotes 
a random variable having this distribution function. The relation between length biasing and the stationary-excess operation is $\widetilde{Y} \stackrel{\mathrm{L}}{=} U \widehat{Y}$, where $U$ has the standard uniform law. See Pakes (1997) and the references therein for these concepts and relations.

Let $X_{\alpha}$ denote a random variable having the limit law in Theorem 3.1, i.e. its LaplaceStieltjes transform is $\psi_{\alpha}(\theta)$.

Theorem 4.1. The law of $X_{\alpha}$ is infinitely divisible and it has the random variable representation

$$
\tilde{X}_{\alpha}=Y_{\alpha}, \text { where } Y_{\alpha}=S_{\alpha}(\gamma(1 / \alpha))^{1 / \alpha},
$$

and its distribution function is

$$
D_{\alpha}(x)=1-\frac{1}{\Gamma(1+1 / \alpha)} \int_{0}^{\infty} y^{-1} \mathrm{e}^{-(x / y)^{\alpha}} \sigma_{\alpha}(y) \mathrm{d} y .
$$

The moment function of $X_{\alpha}$ is

$$
\mathrm{E}\left(X_{\alpha}^{r}\right)=\frac{\alpha \Gamma\left(1+\alpha^{-1}-r / \alpha\right)}{\Gamma(2-r)} \frac{\Gamma(1+r / \alpha)}{\Gamma(1 / \alpha)}, \quad-\alpha<r<\min (1+\alpha, 2) .
$$

Proof. The infinite divisibility assertion will emerge from the proof of Theorem 4.2, below. As observed in Zolotarev (1957),

$$
\int_{0}^{\infty} \mathrm{e}^{-\theta x} \bar{D}_{\alpha}(x) \mathrm{d} x=\left(1+\theta^{\alpha}\right)^{-1 / \alpha} .
$$

Setting $\theta=0$ shows that the first moment of $D_{\alpha}$ is unity, thus preserving in the limit the evident fact that $\mathrm{E}_{1}\left(Z_{t} \bar{F}(t) \mid T>t\right)=1$ for all $t \geq 0$. Thus, the left-hand side of (4.3) is the Laplace-Stieltjes transform of $\widetilde{X}_{\alpha}$. A simple conditioning calculation shows that the right-hand side of (4.3) is the Laplace-Stieltjes transform of $Y_{\alpha}$, whose law is often called a generalized positive Linnik law. This establishes (4.1).

In addition, $\bar{D}_{\alpha}(x)$ is the density function of $Y_{\alpha}$, so

$$
\int_{0}^{x} \bar{D}_{\alpha}(z) \mathrm{d} z=\mathrm{P}\left(S_{\alpha}^{\alpha} \gamma\left(\frac{1}{\alpha}\right) \leq x^{\alpha}\right)=\mathrm{E}\left(\mathrm{P}\left(\gamma\left(\frac{1}{\alpha}\right) \leq\left(\frac{x}{S_{\alpha}}\right)^{\alpha} \mid S_{\alpha}\right)\right) .
$$

Differentiation gives

$$
\bar{D}_{\alpha}(x)=\alpha x^{\alpha-1} \mathrm{E}\left(S_{\alpha}^{-\alpha} g_{1 / \alpha}\left(\left(\frac{x}{S_{\alpha}}\right)^{\alpha}\right)\right),
$$

and (4.2) follows after some further algebra.

Since $Y_{\alpha}=\widetilde{X}_{\alpha}=U \widehat{X}_{\alpha}$ and $\mathrm{E}\left(\widehat{X}_{\alpha}^{r}\right)=\mathrm{E}\left(X_{\alpha}^{r+1}\right)$ (because $\left.\mathrm{E}\left(X_{\alpha}\right)=1\right)$, it follows that

$$
\mathrm{E}\left(X_{\alpha}^{r+1}\right)=\frac{\mathrm{E}\left(Y_{\alpha}^{r}\right)}{\mathrm{E}\left(U^{r}\right)}=(1+r) \mathrm{E}\left(S_{\alpha}^{r}\right) \mathrm{E}\left(\left(\gamma\left(\frac{1}{\alpha}\right)\right)^{r / \alpha}\right) .
$$

The asserted moment formula follows since $\mathrm{E}\left(S_{\alpha}^{r}\right)=\Gamma(1-r / \alpha) / \Gamma(1-r)$ if $r<\alpha$, and $\mathrm{E}\left((\gamma(\ell))^{r}\right)=\Gamma(\ell+r) / \Gamma(\ell)$ if $r>-\ell$.

Remark 4.1. The generalized Linnik law mentioned in the proof extends the standard Linnik law. We note for later reference that this latter law is the law of $\mathcal{L}_{\alpha}:=S_{\alpha}(\gamma(1))^{1 / \alpha}$ and its Laplace-Stieltjes transform is $\left(1+\theta^{\alpha}\right)^{-1}$; see Kotz et al. (2001, Section 4.3). 
Note that (3.1) implies that $D_{\alpha}(0+)=\psi_{\alpha}(\infty)=0$, so the known moment formula

$$
\mathrm{E}\left(S_{\alpha}^{-1}\right)=\frac{\Gamma(1 / \alpha)}{\alpha}=\Gamma\left(1+\frac{1}{\alpha}\right)
$$

follows from (4.2). The case $\alpha=1$ in (4.3) gives the well-known standard exponential limit law in the circumstance where the offspring law has finite variance.

Theorem 3.1 can be expressed as a limit law which is a mixture of exponential laws, and, hence, infinitely divisible (see Steutel and van Harn (2004, p. 331)). Simply observe that, since $V \in \mathcal{R}_{\alpha}$,

$$
V\left(\frac{x}{\bar{F}(t)}\right) \sim x^{\alpha} V\left(\frac{1}{\bar{F}(t)}\right)=x^{\alpha} \rho t .
$$

Consequently, Theorem 3.1, with (4.2), is equivalent to

$$
\lim _{t \rightarrow \infty} \mathrm{P}_{1}\left(\frac{V\left(Z_{t}\right)}{\rho t} \leq z \mid T>t\right)=\frac{1}{\Gamma(1+1 / \alpha)} \int_{0}^{\infty}\left(1-\mathrm{e}^{-z y^{-\alpha}}\right) y^{\alpha-1} \sigma_{\alpha}(y) \mathrm{d} y .
$$

The substitution $v=y^{-\alpha}$ gives the asserted exponential mixture form. The existence of the limit as a nondefective distribution function was observed for the discrete-time case in Nagaev and Wachtel (2007, p. 757).

Let $X_{\alpha, \delta}$ denote a random variable having the Laplace-Stieltjes transform $\psi_{\alpha, \delta}$ specified in (3.5) and distribution function $D_{\alpha, \delta}$. The function $\psi_{\alpha, \delta}$ with arbitrary positive values of $\alpha$ and $\delta$ has been postulated in Porcu et al. (2007) as a candidate variogram for spatio-temporal modelling. For this application, it is necessary to determine for which parameter values $\psi_{\alpha, \delta}$ is completely monotone. This property trivially follows from Theorem 3.2 if $0<\alpha \leq 1$ and $\delta>0$. Porcu et al. (2007) showed complete monotonicity for other parameter configurations, and their results were extended in Berg et al. (2008).

Details in the proof of Theorem 4.1 can be altered to obtain the following representation of the distribution function $D_{\alpha, \delta}$.

Theorem 4.2. If $0<\alpha \leq 1$ and $0<\delta<1$, then

$$
D_{\alpha, \delta}(x)=1-\frac{\alpha}{\Gamma(1-\delta) \Gamma(\delta / \alpha)} \int_{0}^{\infty}\left(\int_{0}^{1} u^{\delta-1}(1-u)^{-\delta} \mathrm{e}^{-(u x / y)^{\alpha}} \mathrm{d} u\right) y^{-\delta} \sigma_{\alpha}(y) \mathrm{d} y,
$$

and this distribution function is infinitely divisible.

Proof. Observe first from (3.5) that $\left(1-\psi_{\alpha, \delta}(\theta)\right) / \theta=\theta^{\delta-1}\left(1+\theta^{\alpha}\right)^{-\delta / \alpha}$. The left-hand side is the Laplace transform of $\bar{D}_{\alpha, \delta}(x)$ and $\theta^{\delta-1}$ is the Laplace transform of $\omega(x)=$ $x^{-\delta} / \Gamma(1-\delta)$. The factor $\left(1+\theta^{\alpha}\right)^{-\delta / \alpha}$ is the Laplace-Stieltjes transform of $\delta_{\alpha}(\gamma(\delta / \alpha))^{1 / \alpha}$, and performing similar calculations as in the proof of Theorem 4.1 shows that its density is

$$
p_{\alpha, \delta}(x)=\frac{\alpha x^{\delta-1}}{\Gamma(\delta / \alpha)} \int_{0}^{\infty} y^{-\delta} \mathrm{e}^{-(x / y)^{\alpha}} \sigma_{\alpha}(y) \mathrm{d} y .
$$

Hence, $\bar{D}_{\alpha, \delta}(x)$ equals the convolution of $\omega(x)$ and $p_{\alpha, \delta}(x)$, and some algebraic manipulation will produce (4.4).

The infinite divisibility follows by expressing $D_{\alpha, \delta}$ as a mixture of exponential laws, as follows. Observe that

$$
\mathrm{e}^{-(u x / y)^{\alpha}}=\mathrm{E}\left(\mathrm{e}^{-(u x / y) S_{\alpha}^{\prime}}\right)
$$

where $S_{\alpha}^{\prime}$ is an independent copy of $S_{\alpha}$. Since $\mathrm{E}\left(S_{\alpha}^{-\delta}\right)=\Gamma(\delta / \alpha) / \alpha \Gamma(\delta)$, it follows from (4.4) 
that

$$
D_{\alpha, \delta}(x)=\frac{\alpha \Gamma(\delta)}{\Gamma(\delta / \alpha)} \mathrm{E}\left(S_{\alpha}^{-\delta}\left(1-\exp \left\{-\frac{x \beta(\delta, 1-\delta) S_{\alpha}^{\prime}}{S_{\alpha}}\right\}\right)\right)=\mathrm{E}\left(1-\mathrm{e}^{-R_{\alpha, \delta} x}\right),
$$

where $S_{\alpha}^{\prime}, S_{\alpha}$, and $\beta(\delta, 1-\delta)$ are independent, and

$$
\mathrm{P}\left(R_{\alpha, \delta} \leq x\right)=\frac{\mathrm{E}\left(S_{\alpha}^{-\delta} ; \beta(\delta, 1-\delta) S_{\alpha}^{\prime} / S_{\alpha} \leq x\right)}{\mathrm{E}\left(S_{\alpha}^{-\delta}\right)} .
$$

This mixture representation for $D_{\alpha, \delta}$ is well defined if $\delta=1$, thus giving the infinite divisibility assertion in Theorem 4.1 .

A direct random variable representation exists for $X_{\alpha, \delta}$ in the case that $0<\delta \leq \alpha \leq 1$. This is based on the hypergeometric identity

$$
F\left(\frac{\delta}{\alpha}, 1 ; 1 ;-\theta^{-\alpha}\right)=\bar{\psi}_{\alpha, \delta}(\theta)=\left(1+\theta^{-\alpha}\right)^{-\delta / \alpha}
$$

The left-hand side equals

$$
F\left(1, \frac{\delta}{\alpha} ; 1 ;-\theta^{-\alpha}\right)=\frac{1}{B(\delta / \alpha, 1-\delta / \alpha)} \int_{0}^{1} u^{(\delta / \alpha)-1}(1-u)^{-\delta / \alpha}\left(1+u \theta^{-\alpha}\right)^{-1} \mathrm{~d} u
$$

These identities can be expressed as

$$
\begin{aligned}
\psi_{\alpha, \delta}(\theta) & =1-\frac{1}{B(\delta / \alpha, 1-\delta / \alpha)} \int_{0}^{1} u^{(\delta / \alpha)-1}(1-u)^{-\delta / \alpha}\left(1+u \theta^{-\alpha}\right)^{-1} \mathrm{~d} u \\
& =\mathrm{E}\left(\frac{\beta(\delta / \alpha, 1-\delta / \alpha)}{\beta(\delta / \alpha, 1-\delta / \alpha)+\theta^{\alpha}}\right) .
\end{aligned}
$$

The quotient inside the brackets is the Laplace-Stieltjes transform of a (randomly) scaled positive Linnik law, and it follows that

$$
X_{\alpha, \delta} \stackrel{\llcorner}{=} S_{\alpha}\left(\frac{\gamma(1)}{\beta(\delta / \alpha, 1-\delta / \alpha)}\right)^{1 / \alpha} .
$$

This leads to the integral representation

$$
\begin{aligned}
D_{\alpha, \delta}(x) & =1-\frac{1}{B(\delta / \alpha, 1-\delta / \alpha)} \int_{0}^{\infty}\left(\int_{0}^{1} u^{(\delta / \alpha)-1}(1-u)^{-\delta / \alpha} \mathrm{e}^{-u(x / y)^{\alpha}} \mathrm{d} u\right) \sigma_{\alpha}(y) \mathrm{d} y \\
& =\mathrm{E}\left(1-\exp \left(-x\left(\beta\left(\frac{\delta}{\alpha}, 1-\frac{\delta}{\alpha}\right)\right)^{1 / \alpha}\left(\frac{S_{\alpha}^{\prime}}{S_{\alpha}}\right)\right)\right),
\end{aligned}
$$

an exponential mixture representation differing from that derived above. If $\alpha=1$ then $X_{1, \delta}=$ $\gamma(1) / \beta(\delta, 1-\delta)$, which obviously is an exponential mixture, and the distribution function simplifies as

$$
D_{1, \delta}(x)=1-\frac{1}{B(\delta, 1-\delta)} \int_{0}^{1} u^{\delta-1}(1-u)^{-\delta} \mathrm{e}^{-u x} \mathrm{~d} u
$$

If $\delta=\alpha$ then the limit law is the Linnik law, $X_{\alpha, \alpha}=S_{\alpha}(\gamma(1))^{1 / \alpha}$. 
The limit laws of Theorems 3.3 and 3.5 for $0<c<\infty$ are related to exponential tilts. If $K(x)$ is a distribution function supported in $[0, \infty)$ and $v>0$, the exponential tilt of $K$ by $v$ is the distribution function

$$
\mathcal{T}_{v} K(x)=\frac{\int_{0}^{x} \mathrm{e}^{-v y} \mathrm{~d} K(y)}{\widehat{K}(v)} .
$$

Thus,

$$
A_{\alpha, c, \delta}(x)=\frac{D_{\alpha, \delta}(x)-\psi_{\alpha, \delta}\left(c^{-1 / \alpha}\right) \mathcal{T}_{c^{-1 / \alpha}} D_{\alpha, \delta}(x)}{1-\psi_{\alpha, \delta}\left(c^{-1 / \alpha}\right)} .
$$

The structure of this limit law takes a more intuitive form if $\delta=\alpha$. If a random variable $Y$ has the distribution function $K$ then $\mathcal{T}_{\nu} Y$ denotes a random variable having the distribution function $\mathcal{T}_{\nu} K$. Let $X_{\alpha, c, \delta}$ denote a random variable having the distribution function $A_{\alpha, c, \delta}$, let $\delta_{0}$ denote the point mass located at 0 , and recall the notation from Remark 4.1.

Theorem 4.3. (i) If $0 \leq c<\infty$ and $\delta=\alpha$, then

$$
X_{\alpha, c, \alpha} \stackrel{\stackrel{L}{=}}{=} \mathcal{L}_{\alpha}+\mathcal{T}_{c^{-1 / \alpha}} \mathcal{L}_{\alpha}^{\prime}+c^{1 / \alpha} V_{\alpha},
$$

where the summands are independent, $\mathcal{L}_{\alpha}^{\prime} \stackrel{\mathrm{L}}{=} \mathcal{L}_{\alpha}, \mathcal{T}_{\infty} \mathcal{L}_{\alpha}^{\prime}:=0$, and

$$
\mathrm{P}\left(V_{\alpha} \leq x\right)= \begin{cases}(\Gamma(1-\alpha))^{-1} \int_{0}^{x} y^{-\alpha-1}\left(1-\mathrm{e}^{-y}\right) \mathrm{d} y & \text { if } 0<\alpha<1, \\ \delta_{0}(x) & \text { if } \alpha=1 .\end{cases}
$$

(ii) If $\delta=\alpha=1$ and $0 \leq c \leq \infty$, then

$$
X_{1, c, 1} \stackrel{\mathrm{L}}{=} \varepsilon+\frac{c}{1+c} \varepsilon^{\prime}
$$

where $\varepsilon$ and $\varepsilon^{\prime}$ are independent and they have the standard exponential law.

Proof. Algebraic manipulation yields

$$
\begin{aligned}
\widehat{A}_{\alpha, c, \alpha}(\theta) & =\left[\frac{\left(\theta+c^{-1 / \alpha}\right)^{\alpha}}{1+\left(\theta+c^{-1 / \alpha}\right)^{\alpha}}-\frac{\theta^{\alpha}}{1+\theta^{\alpha}}\right](1+c) \\
& =\frac{\left(\theta+c^{-1 / \alpha}\right)^{\alpha}-\theta^{\alpha}}{1+\theta^{\alpha}} \frac{1+c}{1+\left(\theta+c^{-1 / \alpha}\right)^{\alpha}} \\
& =\frac{1}{1+\theta^{\alpha}} \frac{1+1 / c}{1+\left(\theta+c^{-1 / \alpha}\right)^{\alpha}}\left[\left(1+c^{1 / \alpha} \theta\right)^{\alpha}-\left(c^{1 / \alpha} \theta\right)^{\alpha}\right]
\end{aligned}
$$

Referring to Theorem 3.1, the first two factors account for $\mathcal{L}_{\alpha}$ and $\mathcal{T}_{c^{-1 / \alpha}} \mathcal{L}_{\alpha}^{\prime}$, respectively. Next,

$$
\mathrm{E}\left(\mathrm{e}^{-\theta V_{\alpha}}\right)=(1+\theta)^{\alpha}-\theta^{\alpha}
$$

which can be seen by inverting two Laplace transforms obtained by differentiating the righthand side.

If $\delta=\alpha=1$ then $\mathrm{P}\left(V_{1}=0\right)=1$, and the Laplace-Stieltjes transform of $\mathcal{T}_{1 / c} \mathcal{L}_{1}$ is $[1+c \theta /(1+c)]^{-1}$, defined as $(1+\theta)^{-1}$ if $c=\infty$. 
Representation (4.5) has long been known in the Galton-Watson context. See Athreya and Ney (1972, p. 61) and Pakes (1999, p. 970) for some commentary. Theorem 3.4 shows that the case $c=\infty$ requires the restriction $\delta=1$. Let $W_{\alpha}$ denote $X_{\alpha, \infty, 1}$. The limiting Laplace-Stieltjes transform in Theorem 3.4 satisfies $\widehat{A}_{\alpha, \infty}(\theta)=-\psi_{\alpha}^{\prime}(\theta)$, the Laplace-Stieltjes transform of the length-biased distribution function $\widehat{D}_{\alpha}$. Hence, $W_{\alpha}=\widehat{X}_{\alpha}$. On the other hand,

$$
\widehat{A}_{\alpha, \infty}(\theta)=\frac{1}{1+\theta^{\alpha}} \frac{\bar{\psi}_{\alpha}(\theta)}{\theta} .
$$

The first factor is the Laplace-Stieltjes transform of $\mathcal{L}_{\alpha}$ and the second factor is the LaplaceStieltjes transform of $\widetilde{X}_{\alpha}$. Hence,

$$
W_{\alpha} \stackrel{\llcorner}{=} \widehat{X}_{\alpha} \stackrel{\llcorner}{=} \mathcal{L}_{\alpha}+\widetilde{X}_{\alpha} .
$$

It follows from Theorem 3.4 and (4.1) that this identity can be expressed as the decomposition

$$
S_{\alpha}\left[\gamma\left(1+\alpha^{-1}\right)\right]^{1 / \alpha} \stackrel{\mathrm{L}}{=} \mathcal{L}_{\alpha}+S_{\alpha}^{\prime}\left[\gamma^{\prime}\left(\alpha^{-1}\right)\right]^{1 / \alpha},
$$

where the prime notation denotes independent copies.

If the norming in Theorem 3.5 is replaced with $\bar{F}(t)$ then the limiting Laplace-Stieltjes transform is $\psi_{\alpha, \delta}\left(\theta+c^{-1 / \alpha}\right) / \psi_{\alpha, \delta}\left(c^{-1 / \alpha}\right)$, i.e. the limiting distribution function is $\mathcal{T}_{c^{-1 / \alpha}} D_{\alpha, \delta}(x)$. This outcome is not entirely satisfactory because this limit converges to $\delta_{0}$ as $c \rightarrow \infty$. In other words, norming with $\bar{F}(t)$ in Theorem 3.6 would overcompensate the growth of $Z_{t}$ under the conditioning $t<T \leq t+o(t)$. On the other hand, norming with $\bar{F}(\tau)$ in Theorem 3.5 in the case $0<c<\infty$ gives the limiting Laplace-Stieltjes transform $\psi_{\alpha, \delta}\left((1+\theta) c^{-1 / \alpha}\right) / \psi_{\alpha, \delta}\left(c^{-1 / \alpha}\right)$, and this tends to 1 as $c \rightarrow \infty$. The norming we have adopted yields limit laws which are nondegenerate throughout the range $0 \leq c \leq \infty$.

The limiting Laplace-Stieltjes transform for any of these normings has the general form $\lambda_{\alpha}(\theta)=\psi_{\alpha, \delta}(a \theta+b) / \psi_{\alpha, \delta}(b)$, where $b=c^{-1 / \alpha}$ and $a=1, a=c^{-1 / \alpha}$, or $a=1+c^{-1 / \alpha}$, according to whether the norming function is $\bar{F}(t), \bar{F}(\tau)$, or $\bar{F}(t+\tau)$, respectively. Theorem 4.2 implies that $\lambda_{\alpha}(\theta)$ is an infinitely divisible Laplace-Stieltjes transform. If $\alpha=1$ then

$$
\lambda_{1}(\theta)=\left(1+\frac{a \theta}{1+b}\right)^{-\delta} \frac{(1+b+a \theta)^{\delta}-(b+a \theta)^{\delta}}{(1+b)^{\delta}-b^{\delta}} .
$$

Hence, the limit law is represented as

$$
Y_{c, \delta} \stackrel{\mathrm{L}}{=} \frac{a}{1+b} \gamma(\delta)+a V_{c, \delta},
$$

where

$$
\mathrm{E}\left(\mathrm{e}^{-\theta V_{c, \delta}}\right)=\frac{\left(1+c^{-1 / \alpha}+\theta\right)^{\delta}-\left(c^{-1 / \alpha}+\theta\right)^{\delta}}{\left(1+c^{-1 / \alpha}\right)^{\delta}-c^{-\delta / \alpha}},
$$

and the corresponding density function is

$$
\left(N x^{1+\delta}\right)^{-1}\left(1-\mathrm{e}^{-x}\right) \exp \left(-c^{-1 / \alpha} x\right), \quad \text { where } \quad N=\Gamma(1-\delta)\left(\left(1+c^{-1 / \alpha}\right)^{\delta}-c^{-\delta / \alpha}\right) .
$$

Note that $a=1+b$ for all $\delta$ with the norming used in Theorem 3.5. This is especially propitious in the case $\alpha=\delta=1$.

Theorem 4.4. If $\alpha=\delta=1$ then the limit law of Theorem 3.5 is the standard exponential for all $c \in[0, \infty]$. 


\section{The case $\alpha=0$}

If (1.1) holds with $\alpha=0$ then

$$
V(x)=\int_{1}^{x} \frac{\mathrm{d} v}{v L(v)}, \quad x \geq 1,
$$

implying that $V \in$ s. In fact, since $f^{\prime \prime}(1-)=\infty$, it follows from (1.1) that $x L(x)$ is unboundedly increasing, implying that $V \in \varsigma_{0}$. Recall that $V(\infty)=\infty$. These facts imply that

$$
V(x)=C \exp \left(\int_{e}^{x} \frac{\varepsilon(v)}{v} \mathrm{~d} v\right),
$$

where $C>0$ is a constant. It follows that the index function

$$
\varepsilon(x)=\frac{x V^{\prime}(x)}{V(x)}=\frac{1}{L(x) V(x)}
$$

is slowly varying and that $L(x) V(x) \rightarrow \infty$. In addition, $\varepsilon(1+)=\infty$, and since $V(1)=0$, we have $\int_{1}^{\mathrm{e}}(\varepsilon(v) / v) \mathrm{d} v=\infty$.

The slow variation of $V$ implies that in representations (2.3) and (2.4), the function $R$ belongs to the class of Karamata rapidly varying functions, $K R_{\infty}$. There is an integral representation for members of $K R_{\infty}$ (see Bingham et al. (1987, Section 2.4)), but in our case this simplifies because $V$ is differentiable in $(1, \infty)$. We will state and prove this in greater generality than we need.

Lemma 5.1. Suppose that $V$ is given by (5.2) with $\varepsilon$ positive and continuous in $(1, \infty)$, and that $V(1)=0$.

(i) Then its rapidly varying inverse $R$ has the representation

$$
R(y)=\exp \left(\int_{0}^{y} \frac{\delta(v)}{v} \mathrm{~d} v\right), \quad y \geq 0,
$$

where $\delta(y)=1 / \varepsilon(R(y)) \rightarrow \infty$ as $y \rightarrow \infty$.

(ii) If (5.1) also holds with $L \in \&$ and continuous in $(1, \infty)$, then

$$
\delta(y)=y L(R(y)) .
$$

Proof. The index function assumptions ensure that $V$ is differentiable and strictly increasing, and its inverse has these properties. Differentiating the identity $V(R(y))=y$ yields

$$
1=R^{\prime}(y) V^{\prime}(R(y))=R^{\prime}(y) V(R(y)) \frac{\varepsilon(R(y))}{R(y)}=R^{\prime}(y) \frac{y \varepsilon(R(y))}{R(y)} .
$$

Hence, $y R^{\prime}(y) / R(y)=\delta(y)$, as defined in the statement. Then (5.4) follows since $R(0)=1$, and (5.5) follows from (5.3).

Remark 5.1. If no assumption is made about $V(1)$ then (5.4) holds after including the multiplicative constant $R(0)$.

The next result establishes a link with von Mises distribution functions. 
Lemma 5.2. If the conditions of Lemma 5.1(ii) hold then, as $y \rightarrow \infty$,

$$
I(z, y)=\int_{y}^{y+z y / \delta(y)} \frac{\delta(v)}{v} \mathrm{~d} v \rightarrow z
$$

locally uniformly with respect to $z \in(-\infty, \infty)$.

Proof. The function $a(y)=y / \delta(y)=1 / L(R(y))$ is differentiable and

$$
-a^{\prime}(y)=\frac{R^{\prime}(y) L^{\prime}(R(y))}{(L(R(y)))^{2}}=\frac{\delta(y)}{y L(R(y))} \frac{R(y) L^{\prime}(R(y))}{L(R(y))}=\frac{R(y) L^{\prime}(R(y))}{L(R(y))} \rightarrow 0,
$$

using (5.5) and $L \in \diamond_{0}$. Rewriting

$$
I(z, y)=\int_{y}^{y+z a(y)} \frac{\mathrm{d} v}{a(v)},
$$

the assertion follows from the proof of Proposition 3.3.25 of Embrechts et al. (1997).

Remark 5.2. Observe that in terms of the notation used in the proof of Lemma 5.2,

$$
G(y)=1-\exp \left(-\int_{0}^{y} \frac{\mathrm{d} v}{a(v)}\right) .
$$

Hence, the proof of Lemma 5.2 amounts to showing that (1.1) with $\alpha=0$ implies that $G$ is a von Mises distribution function (see Embrechts et al. (1997, p. 138)), and, hence, the distribution function $F$ of the time to extinction is a von Mises distribution function too. In particular, $F$ and $G$ lie in the maximal domain of attraction of the Gumbel law (denoted by, e.g. $F \in \mathscr{D}(\Lambda)$ ). Combining details of the last proof and general results about attraction to the Gumbel law shows, under the conditions of Lemma 5.2, that

$$
\lim _{y \rightarrow \infty} \frac{\bar{G}(y+z / L(R(y)))}{\bar{G}(y)}=\mathrm{e}^{-z},
$$

locally uniformly with respect to real valued $z$. See Embrechts et al. (1997, p. 143). If $0<\alpha \leq 1$ then, as we have seen, $R \in \mathcal{R}_{1 / \alpha}$. In this case $G$ lies in the maximal domain of attraction of a Fréchet law. This highlights the difference between the cases $\alpha=0$ and $\alpha>0$.

The next result is our analogue of Theorem 3.1 for $\alpha=0$.

Theorem 5.1. Suppose that (1.1) with $\alpha=0$ and (3.3) with $0<\delta \leq 1$ both hold. Then

$$
\lim _{t \rightarrow \infty} \mathrm{P}_{\pi}\left(L(R(\rho t)) V\left(Z_{t}\right) \leq z \mid Z_{t}>0\right)=1-\mathrm{e}^{-\delta z}, \quad z>0 .
$$

Proof. Let $\theta, z>0$ and

$$
s_{t}=\exp \left(\frac{-\theta}{R(z / L(R(\rho t)))}\right) .
$$

Substituting into (2.3) gives an expression in which $\rho$ and $t$ occur only as the product $\rho t$. Hence we can, and shall, set $\rho=1$ to simplify notation. With reference to (5.4), it follows that

$$
\left(1-s_{t}\right)^{-1} \sim \theta^{-1} R\left(\frac{z t}{\delta(t)}\right)
$$


and, since $V \in \&$,

$$
V\left(\left(1-s_{t}\right)^{-1}\right) \sim V\left(R\left(\frac{z t}{\delta(t)}\right)\right)=\frac{z t}{\delta(t)} .
$$

Hence, (2.3), (2.4), and (5.3) yield

$$
\frac{\bar{F}(t)}{\bar{F}\left(s_{t}, t\right)}=\frac{R(t+(t / \delta(t))(z+o(1)))}{R(t)}=\exp (I(z+o(1), t)) \rightarrow \mathrm{e}^{z},
$$

where we have used Lemma 5.2 for the final step. Consequently,

$$
\lim _{t \rightarrow \infty} \frac{\pi\left(F\left(s_{t}, t\right)\right)-\pi(F(t))}{1-\pi(F(t))}=1-\mathrm{e}^{-\delta z} .
$$

The limit is the Laplace-Stieltjes transform of the defective law which assigns mass $1-\mathrm{e}^{-\delta z}$ at the origin and no mass in $(0, \infty)$. It follows from the continuity theorem for Laplace-Stieltjes transforms (see Feller (1971, p. 431)) that (restoring $\rho$ )

$$
\lim _{t \rightarrow \infty} \mathrm{P}_{\pi}\left(Z_{t} \leq R\left(\frac{x}{L(R(\rho t))}\right) \mid Z_{t}>0\right)=1-\mathrm{e}^{-\delta z},
$$

and the assertion follows.

Remark 5.3. The argument in the last paragraph serves to prove Lemma 1 of Nagaev and Wachtel (2007).

The following result is implied by (5.8). Let $\left(Z_{t}(i): t \geq 0\right)$ denote the MBP obtained as the superposition of $i$ independent copies of $\left(Z_{t}: t \geq 0\right)$ initiated by a single ancestor, $Z_{0}=1$.

Corollary 5.1. Suppose that (1.1) holds with $\alpha=0$, and let $(i(t): t \geq 0$ ) be positive integers satisfying $i(t) \sim c / \bar{F}(t)$, where $c>0$. Then

$$
\lim _{t \rightarrow \infty} \mathrm{P}\left(L(R(\rho t)) V\left(Z_{t}[i(t)]\right) \leq z\right)=\exp \left(-c \mathrm{e}^{-z}\right)
$$

The situation with conditioning events such as $T>t+\tau$ is more delicate in nature than for the case $\alpha>0$. In particular, we have not found a means of interpolating the extreme conditioning events $Z_{t}>0$ and $Z_{\infty}>0$. However, the following result holds.

Theorem 5.2. Assume that the conditions of Theorem 5.1 hold, and let $0<\ell<\infty$. If

$$
\tau \sim \frac{\ell}{\rho L(R(\rho t))}
$$

then

(i) $\lim _{t \rightarrow \infty} \mathrm{P}_{\pi}\left(L(R(\rho t)) V\left(Z_{t}\right) \leq z \mid T>t+\tau\right)=1-\mathrm{e}^{-\delta(z-\ell)^{+}}$;

and

$$
\text { (ii) } \lim _{t \rightarrow \infty} \mathrm{P}_{\pi}\left(L(R(\rho t)) V\left(Z_{t}\right) \leq z \mid t<T \leq t+\tau\right)=\frac{1-\mathrm{e}^{-\delta(z \wedge \ell)}}{1-\mathrm{e}^{-\delta \ell}} \text {. }
$$


Proof. Observe first that $\tau \rightarrow \infty$ and $\tau / t \rightarrow 0$. Just as in the proof of Theorem 5.1, we can and shall set $\rho=1$ without loss of generality.

Noting that the right-hand side of (5.9) equals $\ell t / \delta(t)$, it follows from (2.4), (5.4), and Lemma 5.2 that

$$
\frac{\bar{F}(t+\tau)}{\bar{F}(t)}=\exp (-I(t, \ell+o(1))) \rightarrow \mathrm{e}^{-\ell} .
$$

In addition, (5.9) and Lemma 5.2 imply that

$$
\bar{F}(\tau)=\frac{1}{R(\tau)}=1 / R\left(\frac{\ell+o(1)}{L(R(t))}\right) \sim 1 / R\left(\frac{\ell}{L(R(t))}\right)
$$

Consequently, if $s=s_{t}$ is defined as in the proof of Theorem 5.1 then

$$
1-s_{t} F(\tau)=\frac{\theta+o(1)}{R(z t / \delta(t))}+\frac{1}{R((\ell+o(1)) t / \delta(t))} \sim \begin{cases}\frac{\theta}{R(z t / \delta(t))} & \text { if } z<\ell, \\ \frac{1}{R(\ell t / \delta(t))} & \text { if } z>\ell,\end{cases}
$$

where the asymptotic equivalences arise from the rapid variation of $R$. Since $V \in \&$, we infer that

$$
V\left(\frac{1}{1-s_{t} F(\tau)}\right) \sim \begin{cases}z t \delta(t) & \text { if } z<\ell \\ \ell t / \delta(t) & \text { if } z>\ell\end{cases}
$$

It follows from (5.8) that if $z \neq \ell$ then

$$
\lim _{t \rightarrow \infty} \frac{\bar{F}\left(s_{t} F(\tau), t\right)}{\bar{F}(t)}=\exp (-(z \wedge \ell)) .
$$

Since each side is monotone in $z$, this must hold for $z=\ell$ too. This implies that

$$
\mathrm{E}_{\pi}\left(s_{t}^{Z_{t}} \mid T>t+\tau\right) \rightarrow 1-\mathrm{e}^{-\delta(z-\ell)^{+}},
$$

and assertion (i) follows from the argument ending the proof of Theorem 5.1. Assertion (ii) follows in a similar manner.

Remark 5.4. The limit distribution function in Theorem 5.2(i) is that of $\ell+\gamma(1) / \delta$; equivalently, from the lack of memory property, it is the conditional distribution function of $\gamma(1) / \delta$ given that $\gamma(1) / \delta>\ell$. Dually, the limit distribution function in Theorem 5.2(ii) is the conditional distribution function of $\gamma(1) / \delta$ given that $\gamma(1) / \delta \leq \ell$.

Clearly, the limit law in Theorem 5.2(i) tends to the point mass at infinity as $\ell \rightarrow \infty$, suggesting that the norming used for Theorem 5.2 is not appropriate for the conditioning $Z_{\infty}>0$. Our next result verifies this intuition.

Theorem 5.3. Suppose that (1.1) holds with $\alpha=0$, and that $\pi$ is an initial law with finite first moment. Then

$$
\lim _{t \rightarrow \infty} \mathrm{P}_{\pi}\left(\rho t L\left(Z_{t}\right) \leq z \mid Z_{\infty}>0\right)=1-\mathrm{e}^{-z}, \quad z>0 .
$$


Proof. We will again set $\rho=1$ for this proof. Defining $w=V\left((1-s)^{-1}\right)$, it follows from (2.4) and the definition of $G$ in Remark 5.2 that $1-s=\bar{G}(w)$. It then follows from (2.3) that

$$
\frac{1-F(s, t)}{1-s}=\frac{\bar{G}(w+t)}{\bar{G}(w)} .
$$

The assumptions $m=1$ and $\alpha=0$ imply that $L(x)$ decreases from $L(1)=f(0)>0$ to $L(\infty-)=0$. Consequently, the function $\lambda(x):=1 / L(x)$ is strictly increasing to $\infty$ in $[1, \infty)$ and slowly varying. Its inverse function, denoted by $r(y)$, increases from unity to infinity on the interval $[1 / f(0), \infty)$. For arbitrary $\theta, z>0$, set

$$
s_{t}=G(w)=\exp \left(-\frac{\theta}{r(t / z)}\right) .
$$

Solving this relation for $t$ as a function of $w$, we obtain

$$
t=z \lambda\left(\frac{-\theta}{\log G(w)}\right) \sim z \lambda\left(\frac{1}{\bar{G}(w)}\right)=z \lambda(R(w))=\frac{z}{L(R(w))} .
$$

It follows from (5.6) and (5.10) that

$$
\lim _{t \rightarrow \infty} \frac{1-F\left(s_{t}, t\right)}{1-s_{t}}=\mathrm{e}^{-z}
$$

independently of $\theta$.

Since $a(s)=(1-s) L\left((1-s)^{-1}\right)$, we conclude from (3.12) that

$$
\lim _{t \rightarrow \infty} \mathrm{E}_{\pi}\left(s_{t}^{Z_{t}} \mid Z_{\infty}>0\right)=\mathrm{e}^{-z}
$$

So, after restoring the presence of $\rho$, then, again as in the proof of Theorem 5.1, we find that

$$
\lim _{t \rightarrow \infty} \mathrm{P}_{\pi}\left(Z_{t} \leq r\left(\frac{\rho t}{z}\right) \mid Z_{\infty}>0\right)=\mathrm{e}^{-z} .
$$

The assertion follows since $Z_{t} \leq r(\rho t / z)$ if and only if $\rho t L\left(Z_{t}\right) \geq z$.

\section{Converse assertions}

We begin with a converse to Theorem 3.1. The proof takes advantage of the additional structure of the MBP. The notation $\stackrel{\text { W }}{\rightarrow}$ ' denotes weak (or vague) convergence of distribution functions.

Theorem 6.1. Let $m=1$, and assume that

$$
H(x, t):=\mathrm{P}_{1}\left(\bar{F}(t) Z_{t} \leq x \mid Z_{t}>0\right) \stackrel{\mathrm{w}}{\rightarrow} H(x),
$$

a nondegenerate distribution function. Then (1.1) holds with $0<\alpha \leq 1$ and $L \in \mathcal{S}$.

Proof. Assumption (6.1) is equivalent to

$$
\lim _{t \rightarrow \infty} \int_{0}^{\infty} g(x) \mathrm{d} H(x, t)=\int_{0}^{\infty} g(x) \mathrm{d} H(x)
$$

for continuous functions $g$ satisfying $\lim _{x \rightarrow \infty} g(x)=0$. Taking $g(x)=\mathrm{e}^{-\theta x}$ and $s_{t}=\mathrm{e}^{-\theta \bar{F}(t)}$ 
yields

$$
\lim _{t \rightarrow \infty} \frac{\bar{F}\left(s_{t}, t\right)}{\bar{F}(t)}=\bar{\psi}(\theta),
$$

where $\psi$ is the Laplace-Stieltjes transform of the distribution function $H$. Taking $g(x)=x \mathrm{e}^{-\theta x}$ yields

$$
\lim _{t \rightarrow \infty} F^{\prime}\left(s_{t}, t\right)=\bar{\psi}^{\prime}(\theta)
$$

Referring to (2.5) and setting $b(u)=a(1-u)$, it follows from (6.3) that

$$
\lim _{t \rightarrow \infty} \frac{b\left(\bar{F}\left(s_{t}, t\right)\right)}{b\left(1-s_{t}\right)}=\bar{\psi}^{\prime}(\theta) .
$$

Observe that $\lambda(\theta)=\bar{\psi}(\theta) / \theta=\int_{0}^{\infty} \mathrm{e}^{-\theta x} \bar{H}(x) \mathrm{d} x$ is decreasing. Since $1-s_{t} \sim \theta \bar{F}(t)$, then setting $u=1-s_{t}$ and expressing (6.2) as $\bar{F}\left(s_{t}, t\right)=u \lambda(\theta)(1+o(1)),(6.4)$ takes the form

$$
\lim _{u \rightarrow 0} \frac{b(\lambda(\theta) u(1+o(1)))}{b(u)}=\bar{\psi}^{\prime}(\theta)=\xi(\lambda(\theta))
$$

for some function $\xi$. Standard arguments using the continuity and monotonicity of $b(u)$ imply that $\lim _{u \rightarrow 0} b(\lambda u) / b(u)=\xi(\lambda)$ for all $0<\lambda<\lambda(0+)$, and, hence, that $b(u)$ is regularly varying at 0 , i.e. $b(u)=u^{r} L(1 / u)$, where $r \geq 0$ and $L \in \delta$. This conclusion is equivalent to (1.1) with $\alpha=r-1$. Since $(f(s)-s) /(1-s)=(1-s)^{\alpha} L(1 / s)$, it follows that $0 \leq a \leq 1$.

In addition, $\xi(\lambda)=\lambda^{1+\alpha}$, so

$$
\left(\frac{\bar{\psi}(\theta)}{\theta}\right)^{1+\alpha}=\bar{\psi}^{\prime}(\theta) .
$$

If $\alpha=0$, this has the solution $\psi(\theta)=1-K \theta$ for some constant $K$. But this is the LaplaceStieltjes transform of a distribution function only if $K=0$, in which case $H$ is the point mass at 0 . Hence, $\alpha>0$, thus proving the assertion.

Remark 6.1. As its discrete-time counterpart (see Slack (1972)), Theorem 6.1 leaves open the question of whether (1.1) is implied by the more general conditional limit $\mathrm{P}_{1}\left(v(t) Z_{t} \leq\right.$ $\left.x \mid Z_{t}>0\right) \stackrel{\mathrm{W}}{\rightarrow} H(x)$, where $v(t)>0$ and $v(t) \rightarrow 0$. If $s_{t}=\exp (-\theta v(t))$ then the initial steps of the above proof lead to the generalized form of (6.4) as

$$
\lim _{t \rightarrow \infty} \frac{v(t)}{\bar{F}(t)} \frac{b\left(\bar{F}\left(s_{t}, t\right)\right)}{b\left(1-s_{t}\right)}=\bar{\psi}^{\prime}(\theta) .
$$

It follows immediately that $v(t) / \bar{F}(t)$ must be bounded away from 0 , for otherwise the limit is identically 0. Similarly, the convergence of types theorem (see Feller (1971, p. 253)) implies that if (1.1) does hold then $v(t) / \bar{F}(t)$ has a positive and finite limit. So, conversely, if $v\left(t_{n}\right) / \bar{F}\left(t_{n}\right) \rightarrow$ $\infty$ as $t_{n} \rightarrow \infty$ then (1.1) cannot hold.

Theorem 6.1 extends to a converse for initial laws, as follows. Let $\mathscr{D}_{I}$ denote initial laws $\pi$ such that

$$
\lim _{t \rightarrow \infty} \mathrm{P}_{\pi}\left(\bar{F}(t) Z_{t} \leq x \mid Z_{t}>0\right)=H_{\pi}(x)
$$

where the limit is a nondegenerate distribution function. Recall that $m_{\pi}$ is the first moment of $\pi$. 
Theorem 6.2. If $\mathcal{D}_{I}$ contains an initial law with $m_{\pi}<\infty$ then the conclusion of Theorem 6.1 holds and (3.3) holds with $0<\delta \leq 1$ and $\mathcal{L} \in$ \&.

Proof. If $p(u)=1-\pi(1-s)$ and $s_{t}$ is as defined in the previous proof, then (6.5) is equivalent to

$$
\lim _{t \rightarrow \infty} \frac{p\left(\bar{F}\left(s_{t}, t\right)\right)}{p(\bar{F}(t))}=\widehat{H}_{\pi}(\theta) .
$$

If $m_{\pi}<\infty$, the argument of the limit operation is asymptotically proportional to $\bar{F}\left(s_{t}, t\right) / \bar{F}(t)$, and, hence, the conditions of Theorem 6.1 are satisfied, and its conclusion holds. It follows that $\mathscr{D}_{I}$ contains all initial laws with finite first moment, and for these, the limit distribution function is independent of $\pi$, i.e. $\widehat{H}_{\pi}(\theta)=\psi_{\alpha}(\theta)$.

If $\pi \in \mathscr{D}_{I}$ and $m_{\pi}=\infty$, then arguing as in the proof of Theorem 6.1, now with $u=\bar{F}(t)$, it follows from (6.2) and (6.6) that

$$
\lim _{u \rightarrow 0} \frac{p\left(\bar{\psi}_{\alpha}(\theta) u(1+o(1))\right)}{p(u)}=\widehat{H}_{\pi}(\theta)=\xi\left(\bar{\psi}_{\alpha}\right) .
$$

This implies that (3.3) holds, and $\delta$ must be positive since $H_{\pi}$ is nondegenerate.

Remark 6.2. The proof still holds if the hypotheses are broadened a little by assuming that $\mathcal{D}_{I}$ contains at least one initial law having the form (3.3) with $\mathcal{L} \in \delta$. This would imply that all the limit points of $\bar{F}\left(s_{t}, t\right) / \bar{F}(t)$ as $t \rightarrow \infty$ equal $\widehat{H}_{\pi}(\theta)$. Theorem 6.1 is then applicable, and the rest follows.

Assume now that $\alpha=0$ in (1.1), and that $L$ decreases to 0 . Differentiating (1.1) expressed as

$$
L\left(\frac{1}{u}\right)=\frac{f(1-u)}{u}-\frac{1}{u}+1
$$

shows that $L^{\prime \prime}(1 / u)=u^{3} f^{\prime \prime}(1-u)$, and, hence, that $L$ is convex decreasing. Part (i) of the next result is only a partial converse to Theorem 5.1, though it is not obvious how to improve it. Part (ii) is a complete converse to the domain of attraction outcome discussed in Remark 5.2.

Theorem 6.3. Let $L(x)>0(x>0)$ satisfy $L(\infty)=0$, and let $s_{t}(\theta)$ be defined by (5.7).

(i) Suppose for $0<\epsilon<1$ that

$$
\lim _{t \rightarrow \infty} \frac{\bar{F}\left(s_{t}(\theta), t\right)}{\bar{F}(t)}=B(z)
$$

exists for all $z \geq 0$ and $\theta \in(1-\epsilon, 1+\epsilon)$, where $B$ is independent of $\theta$ and nondegenerate. Then (1.1) holds with $\alpha=0$ and $L \in \delta_{0}$, and $B(z)=\mathrm{e}^{-z}$.

(ii) If $F \in \mathscr{D}(\Lambda)$ then $L \in \varsigma_{0}$, and, hence, $F$ is a von Mises distribution function.

Proof. (i) Set $\rho=1$, as we have previously done. The assumption $L(\infty)=0$ implies that $1-s_{t}(\theta) \sim \theta / R(z / L(R(t)))$, and, hence, we can choose $1-\epsilon<\theta_{1}<1<\theta_{2}<1+\epsilon$ such that

$$
\frac{1}{1-s_{t}\left(\theta_{1}\right)}<R\left(\frac{z}{L(R(t))}\right)<\frac{1}{1-s_{t}\left(\theta_{2}\right)} .
$$


It follows from (2.3), (2.4), and (6.7) that the distribution function $G(t)=1-1 / R(t)$ satisfies (cf. (5.6))

$$
\lim _{t \rightarrow \infty} \frac{\bar{G}(t+z / L(R(t)))}{\bar{G}(t)}=B(z) .
$$

Setting $t=V(x)$, this outcome is equivalent to

$$
\lim _{x \rightarrow \infty} x \bar{G}\left(V(x)+\frac{z}{L(x)}\right)=B(z) .
$$

This condition is necessary and sufficient for $G$ to be in the maximal domain of attraction of an extreme value law, which is either a Fréchet law or the Gumbel law. In the Fréchet case, $B(z) \propto z^{-\kappa}$ for some $\kappa>0$. It follows from extreme value theory (see Embrechts et al. (1997, p. 131)) that $R \in \mathcal{R}_{\kappa}$. It can be argued from (2.4) that this outcome implies that (1.1) holds with $\alpha=1 / \kappa$ and $L$ slowly varying, and, hence, that $B(z)$ is not independent of $\theta$.

So it follows that $G$ is attracted to a Gumbel law, and, in particular, that $B(z)=\mathrm{e}^{-\mu z}$, where $\mu>0$ is a scale constant. There is a possible translation parameter, but its value is 0 because $B(0)=1$. So condition (6.8) is equivalent to

$$
\lim _{t \rightarrow \infty} \frac{R(t+z / \mu L(R(t)))}{R(t)}=\mathrm{e}^{z} .
$$

This defines membership of a class of functions, denoted by $\Gamma$ in Bingham et al. (1987, p. 175), and there is an associated auxiliary function, $g(y)=1 / \mu L(R(y))$. Membership in $\Gamma$ has several consequences of which the most relevant is that $g(V(x))=1 / \mu L(x) \in \delta$, i.e. $L \in \delta$; see Bingham et al. (1987, Theorem 3.10.4). It follows from (1.1) that $L \in \varsigma_{0}$, and Theorem 5.1 implies that $\mu=1$.

(ii) The necessary and sufficient condition for $F \in \mathscr{D}(\Lambda)$ (see Embrechts et al. (1997, p. 143)) can be expressed in terms of $R(y)$ as the existence of a function $g(y) \rightarrow \infty$ such that

$$
\lim _{y \rightarrow \infty} \frac{R(y+z g(y))}{R(y)}=\mathrm{e}^{z}, \quad-\infty<z<\infty .
$$

As above, this is equivalent to $R \in \Gamma$ and $\ell(x):=g(V(x)) \in 8$, implying that $k(x):=$ $x / \ell(x) \in \mathcal{R}_{1}$. In addition, we may choose (see Embrechts et al. (1997, Equation (3.34)))

$$
g(y)=R(y) \int_{y}^{\infty} \frac{\mathrm{d} v}{R(v)},
$$

so

$$
\ell(x)=x \int_{x}^{\infty} \frac{\mathrm{d} w}{w^{2} L(w)},
$$

using the substitution $w=R(v)$.

Since $k^{\prime}(x)$ exists, we have $x k^{\prime}(x) / k(x) \rightarrow 1$. Computation shows this is equivalent to $x \ell^{\prime}(x) / \ell(x) \rightarrow 0$, and since

$$
\ell^{\prime}(x)=\frac{\ell(x)}{x}-\frac{1}{\ell(x) L(x)},
$$

we conclude that $\ell(x) L(x) \rightarrow 1$, i.e. $L \in \delta$. Finally, since $L$ is convex, it follows from Lamperti's theorem that $L \in \varsigma_{0}$. 
Identifying the centring and norming sequences for $T$ from the general expressions in Theorem 3.3.26 of Embrechts et al. (1997) and using (2.4) leads to the following companion of Theorem 3.7.

Theorem 6.4. Suppose that (1.1) with $\alpha=0$ and (3.3) with $0<\delta \leq 1$ both hold. Then

and

$$
\text { (i) } \lim _{t \rightarrow \infty} \mathrm{P}_{\pi}\left(L\left(\frac{1}{\bar{F}(t)}\right)(T-t) \leq z \mid T>t\right)=1-\mathrm{e}^{-\delta z}, \quad z \geq 0 \text {; }
$$

(ii) $\lim _{i \rightarrow \infty} \mathrm{P}_{i}(L(i)(\rho T-V(i)) \leq z)=\exp \left(-\mathrm{e}^{-z}\right), \quad-\infty<z<\infty$.

\section{Discrete limit laws}

We begin by discussing the asymptotic behaviour of the invariant measure $\left\{\mu_{j}: j \geq 1\right\}$ (recall (2.2)) in relation to (1.1). If $g_{j}=\sum_{i>j} p_{i}$ then

$$
g(s)=\sum_{j=0}^{\infty} g_{j} s^{j}=\frac{1-f(s)}{1-s}
$$

is a PGF. It follows from (2.2) that $\mathcal{M}^{\prime}(s)=U(s) /(1-s)$, where

$$
U(s)=\sum_{j=0}^{\infty} u_{j} s^{j}:=\frac{1}{1-g(s)} .
$$

Thus, $\left\{u_{j}: j \geq 0\right\}$ is a renewal sequence. Its generating law $\left\{g_{j}\right\}$ is nonincreasing in $j$ and, hence, has a unit maximal span. These relations imply that the invariant measure has the representation

$$
\mu_{j}=j^{-1} \sum_{i=0}^{j-1} u_{i}, \quad j \geq 1 .
$$

Theorem 7.1. (i) If $m=1$ then the sequence $\left\{j \mu_{j}\right\}$ is nondecreasing and $\mu_{j+1} \sim \mu_{j}$.

(ii) Suppose that $0 \leq \alpha \leq 1$ and $L \in$ s. Then (1.1) is equivalent to

$$
\mu_{j} \sim \frac{1}{\Gamma(1+\alpha) j^{1-\alpha} L(j)} .
$$

Proof. Assertion (i) follows from (7.1). For (ii), just observe that the generating function of $\left\{j \mu_{j}\right\}$ is $1 /(f(s)-s)$, and that a Tauberian theorem for power series (see Bingham et al. (1987, p. 40)) asserts that, since $j \mu_{j}$ is monotone, (1.1) is equivalent to $j \mu_{j} \sim j^{\alpha} / \Gamma(1+\alpha) L(j)$ : with $\rho$ as denoted in Corollary 1.7.3 of Bingham et al. (1987), set $\rho=1+\alpha$. The last asymptotic relation is the same as (7.2).

Remark 7.1. (i) If $\sigma^{2}=f^{\prime \prime}(1-)<\infty$ then $\alpha=1$ and $L(\infty)=\frac{1}{2} \sigma^{2}$, so $\mu_{j} \rightarrow 2 / \sigma^{2}$. This one-way implication was first shown in Yang (1973). The converse is new.

(ii) Slack (1968, Theorem 2) proved a global version of the local asymptotic equivalence (7.2) for the Galton-Watson process in the case $0<\alpha \leq 1$. To the author's knowledge, no corresponding local version is known, except in the case where $f^{\prime \prime}(1)$ is finite. See Pommerenke (1981) for this result. 
In what follows we assume only that $m=1$. It follows from (2.5) that, since the right-hand side is monotone in $t$,

$$
\lim _{s \rightarrow 1} \frac{a(F(s, t))}{a(s)}=1,
$$

locally uniformly with respect to $t$. This, together with $F(F(t), \tau)=F(t+\tau)$, implies that

$$
\lim _{t \rightarrow \infty} \frac{a(F(t+\tau))}{a(F(t))}=1,
$$

locally uniformly with respect to $\tau$.

Lemma 7.1. If $m=1$ and $u, v>0$, then

$$
\lim _{t \rightarrow \infty} \frac{F(t+u)-F(t)}{F(t+v)-F(t)}=\frac{u}{v} .
$$

Proof. It follows from the mean value theorem, (2.5), and (7.3) that, for some $\zeta_{t} \in(t, t+u)$,

$$
\frac{F(t+u)-F(t)}{a(F(t))}=u \frac{a\left(F\left(t+\zeta_{t}\right)\right)}{a(F(t))} \rightarrow u,
$$

and the assertion follows.

Let $B$ be a Borel subset of the positive reals and denote its Lebesgue measure by $|B|$. Recalling that $F(t)$ is a distribution function, let $F(B)$ denote the probability mass assigned to $B$ by $F$, and let $B_{F}=\{u=F(t): t \in B\}$ denote the image of $B$ under $F$. In the case that $B$ is a bounded interval, the next result is the MBP analogue of the principal result in Seneta (1967). Seneta's proof is based on the functional iteration theory associated with the Abel functional equation. The MBP setting permits a direct and self-contained approach. Recall that $T$ denotes the extinction time.

Theorem 7.2. If $m=1$ and $m_{\pi}<\infty$, then

$$
\lim _{t \rightarrow \infty} \mathrm{P}_{\pi}\left(Z_{t}=j \mid T \in t+B\right)= \begin{cases}\mu_{j} \int_{B_{F}} \mathrm{~d} u^{j} / \rho|B| & \text { if }|B|<\infty, \\ 0 & \text { if }|B|=\infty .\end{cases}
$$

Proof. Since

$$
\mathrm{P}_{j}(T \in B)=\int_{B} \mathrm{~d} F^{j}(v)=j \int_{B} F^{j-1}(v) a(F(v)) \mathrm{d} v,
$$

it follows that

$$
\begin{aligned}
\mathrm{E}_{\pi}\left(s^{Z_{t}} ; T \in t+B\right) & =\sum_{j \geq 1} p_{\pi, j}(t) s^{j} \mathrm{P}_{j}(T \in B) \\
& =\left.s \int_{B} \frac{\partial}{\partial u} \pi(F(u, t))\right|_{u=s F(v)} a(F(v)) \mathrm{d} v \\
& =s \int_{B} \pi^{\prime}(F(s F(v), t)) \frac{a(F(s F(v), t))}{a(s F(v))} a(F(v)) \mathrm{d} v .
\end{aligned}
$$


However, since $a$ is decreasing,

$$
\frac{a(F(t+v))}{a(F(t))} \leq \frac{a(F(s F(v), t))}{a(F(t))} \leq 1,
$$

so it follows from (7.3) and the dominated convergence theorem that

$$
\lim _{t \rightarrow \infty} \frac{\mathrm{E}_{\pi}\left(s^{Z_{t}} ; T \in t+B\right)}{a(F(t))}=m_{\pi} s \int_{B} \frac{a(F(v))}{a(s F(v))} \mathrm{d} v,
$$

provided that the integral (denoted by $I(s, B))$ is finite. Note first that $I(1, B)=|B|$. If $s<1$ then the change of variable $u=F(v)$, implying that $\mathrm{d} u=a(F(v)) \mathrm{d} v$, yields the bound

$$
I(s, B)<\frac{1}{a(s)} \int_{B} a(F(v)) \mathrm{d} v=\frac{1}{a(s)} \int_{B_{F}} \mathrm{~d} u=\frac{\left|B_{F}\right|}{a(s)}<\infty
$$

for any admissible $B$. In fact, since $\mathrm{d}_{u} \mathcal{M}(s u)=s \mathcal{M}^{\prime}(s u) \mathrm{d} u=\rho s \mathrm{~d} u / a(s u)$, we obtain the evaluation

$$
s I(s, B)=\rho^{-1} \int_{B_{F}} \mathrm{~d}_{u} \mathcal{M}(s u)=\rho^{-1} \sum_{j \geq 0} s^{j} \mu_{j} \int_{B_{F}} \mathrm{~d} u^{j},
$$

and the assertion follows if $|B|<\infty$. If $|B|=\infty$ then Fatou's lemma implies that

$$
\liminf _{t \rightarrow \infty} \frac{\mathrm{P}_{\pi}(T \in t+B)}{a(F(t))} \geq m_{\pi} \int_{B} \mathrm{~d} v=\infty .
$$

Since $I(s, B)$ is finite, this outcome implies that $\mathrm{E}_{\pi}\left(s^{Z_{t}} \mid T \in t+B\right) \rightarrow 0$, and the assertion follows in this case.

In the particular case that $B$ is a union of disjoint intervals, $B=\bigcup_{n}\left(a_{n}, b_{n}\right]$, and $|B|<\infty$, then the assertion takes the form

$$
\lim _{t \rightarrow \infty} \mathrm{P}_{\pi}\left(Z_{t}=j \mid T \in t+B\right)=\frac{\mu_{j} \sum_{n}\left(F^{j}\left(b_{n}\right)-F^{j}\left(a_{n}\right)\right)}{\rho|B|} .
$$

\section{Further remarks}

The term 'smooth structure' used in the introduction refers to the basic integral representations in Section 2, and the ease with which they lead to the fundamental results in Sections 3 and 5. We have only to contrast the proofs there with the longer and more intricate proofs used in Slack (1968), (1972) and Nagaev and Wachtel (2007) for the simple branching process (SBP). Although a representation analogous to (2.3) exists in this case, it is not accompanied by a representation such as (1.2) for what in essence is the generating function (2.2) of the invariant measure of the MBP transition semigroup. On the other hand, Borovkov (1989) described a methodology which is applicable to both continuous- and discrete-time (nonhomogeneous) Markov processes. He showed how the results of Slack (1968), (1972) can more easily be derived.

Suppose that $\left(Z_{t}\right)$ denotes either the $\operatorname{MBP}(t \geq 0)$ or $\operatorname{SBP}(t=0,1, \ldots)$, let $F(t)$ denote the extinction time distribution function of either process, and suppose that $m \leq 1$. Borovkov assumed that

$$
\lim _{t \rightarrow \infty} \frac{\bar{F}(t-0)}{\bar{F}(t)}=1 .
$$


This is satisfied by the MBP if $m \leq 1$, and by the SBP only if $m=1$. Indeed (in this paragraph only), let $T_{S}$ denote the extinction time of the SBP, and suppose that $\rho=1$ for the MBP. If $m=1$ and (1.1) holds, then it follows from results of Slack/Borovkov in the case $\alpha>0$, and by piecing together results in Nagaev and Wachtel (2007) in the case $\alpha=0$, that $T_{S}$ and $T$ are tail equivalent, i.e. $\mathrm{P}\left(T_{S}>t\right) / \mathrm{P}(T>t) \rightarrow 1$ as $t \rightarrow \infty$. This implies that both extinction time laws are attracted to the same extreme value law. This is not the case if $m<1$, for then $F(t)$ is attracted to the Gumbel law, but $\mathrm{P}\left(T_{S} \leq t\right)$ is not attracted to any extreme value law; see Pakes (1989).

Continuing our discussion of Borovkov's methodology, for $0<s<1$, let $U(s)=$ $\inf \{t: \bar{F}(t) \leq s\}$ and

$$
q(t, \theta)=\frac{\bar{F}(t+U(\theta A(t)))}{\bar{F}(t)},
$$

where $A(t)$ is positive valued and $A(t) \rightarrow 0$. Then Theorem 1 of Borovkov (1989) states that

$$
\psi(\theta):=\lim _{t \rightarrow \infty} \mathrm{E}\left(\mathrm{e}^{-\theta A(t) Z_{t}} \mid Z_{t}>0\right)
$$

exists if and only if

$$
\lim _{t \rightarrow \infty} q(t, \theta)=1-\psi(\theta) .
$$

This makes clear the relation between limit theorems for the critical branching process and maximal domain of attraction properties of $F(t)$.

Note that the condition $A(t) \rightarrow 0$ removes the subcritical MBP from the ambit of the strict statement of Borovkov's theorem. However, as he indicated (see the bottom of page 106 in Borovkov (1989) for the SBP), a step in his proof encompasses this case. In fact, since $\bar{F}(U(s))=s$, his proof in essence is that

$$
\mathrm{E}\left((1-s)^{Z_{t}} \mid Z_{t}>0\right)=1-\frac{\bar{F}(t+U(s))}{\bar{F}(t)} \rightarrow 1-\exp \left[-(1-m) V\left(\frac{1}{1-s}\right)\right] .
$$

It is worth reporting how Borovkov's (1989) approach to the converse result in Slack (1972) applies with little change to the MBP. In a nutshell, (6.1) is equivalent to the altered form of (6.2),

$$
\lim _{t \rightarrow \infty} \frac{\bar{F}(1-\theta \bar{F}(t))}{\bar{F}(t)}=\bar{\psi}(\theta), \quad \theta>0,
$$

i.e. (cf. (8.1))

$$
q(\theta, t)=\frac{R(\rho t)}{R\left[\rho t+V\left(\theta^{-1} R(\rho t)\right)\right]} \rightarrow \bar{\psi}(\theta) .
$$

Let $\lambda_{j}(t)=\bar{F}(j t) / \bar{F}(t)$. Setting $\theta=1$ in (8.2) yields $q(1, t)=\lambda_{2}(t) \rightarrow \bar{\psi}(1) \in(0,1)$. If $\lambda_{j}(t) \rightarrow \lambda_{j} \in(0,1)$ then the local uniform convergence with respect to $\theta$ in (8.2) implies that

$$
\lambda_{j+1}(t)=q\left(\lambda_{j}(t), t\right) \rightarrow \lambda_{j+1}=\bar{\psi}\left(\lambda_{j}\right)
$$

It follows by induction that $\lim _{t \rightarrow \infty} \bar{F}(\lambda t) / \bar{F}(t) \in(0,1]$ exists if $\lambda$ is a positive integer. This extends to rational valued $\lambda$, and since $\bar{F}$ is monotone, it follows from a theorem of Karamata (see Bingham et al. (1987, p. 54)) that $\bar{F}$ is regularly varying. The conclusion of Theorem 6.1 follows from Remark 3.1. This route to (1.1) is not quite as direct as the proof of Theorem 6.1. 


\section{Acknowledgement}

I thank the anonymous referee for a careful reading of the manuscript and for giving substance to Theorem 6.3.

\section{References}

Anderson, W. J. (1991). Continuous-Time Markov Chains. Springer, New York.

Athreya, K. B. AND Ney, P. E. (1972). Branching Processes. Springer, New York.

Berg, C., Mateu, J. And Porcu, E. (2008). The Dagum family of isotropic correlation functions. Bernoulli 14, 1134-1149.

Bingham, N. H., Goldie, C. M. And Teugels, J. L. (1987). Regular Variation. Cambridge University Press.

Borovkov, K. A. (1989). A method of proving limit theorems for branching processes. Theory Prob. Appl. 33, $105-113$.

Conner, H. (1967). A note on limit theorems for Markov branching processes. Proc. Amer. Math. Soc. 18, $76-86$.

Durrett, R. (1978). Conditioned limit theorems for some null recurrent Markov processes. Ann. Prob. 6, $798-828$.

Embrechts, P., KlüPPelberg, C. AND Mikosch, T. (1997). Modelling Extremal Events. Springer, Berlin.

Feller, W. (1971). An Introduction to Probability Theory and Its Applications, Vol. II, 2nd edn. John Wiley, New York.

Harris, T. E. (1963). The Theory of Branching Processes. Springer, Berlin.

Kotz, S., Kozubowski, T. J. And Podgórski, K. (2001). The Laplace Distribution and Generalizations. Birkhäuser, Boston, MA.

Nagaev, S. V. And WaChTEL, V. (2007). The critical Galton-Watson process without further power moments. J. Appl. Prob. 44, 753-769.

Pakes, A. G. (1989). On the asymptotic behaviour of the extinction time of the simple branching process. Adv. Appl. Prob. 21, 470-472.

PAKes, A. G. (1997). Characterization by invariance under length-biasing and random scaling. J. Statist. Planning Infer. 63, 285-310.

PAKes, A. G. (1999). Revisiting conditional limit theorems for the mortal simple branching process. Bernoulli 5, 969-998.

Pommerenke, C. (1981). On the stationary measures of critical branching processes. Z. Wahrscheinlichkeitsth. 55, 305-312.

Porcu, E., Mateu, J., Zini, A. ANd PINI, R. (2007). Modelling spatio-temporal data: a new variogram and covariance structure proposal. Statist. Prob. Lett. 77, 83-89.

Seneta, E. (1967). The Galton-Watson process with mean one. J. Appl. Prob. 4, 489-495.

Shanbhag, D. N. AND SReehari, M. (1977). On certain self-decomposable distributions. Z. Wahrscheinlichkeitsth. 38, 217-222.

SLACK, R. S. (1968). A branching process with mean one and possibly infinite variance. Z. Wahrscheinlichkeitsth. 9, 139-145.

SLACK, R. S. (1972). Further notes on branching processes with mean 1. Z. Wahrscheinlichkeitsth. 25, 31-38.

Steutel, F. W. And van Harn, K. (2004). Infinite Divisibility of Probability Distributions on the Real Line. Marcel Dekker, New York.

Vatutin, V. A., Wachtel, W. and Fleischmann, K. (2008). Critical Galton-Watson processes: the maximum of total progenies within a large window. Theory Prob. Appl. 52, 470-492.

YANG, Y. S. (1973). Asymptotic properties of the stationary measure of a Markov branching process. J. Appl. Prob. 10, 447-450.

Zolotarev, V. M. (1957). More exact statements of several theorems in the theory of branching processes. Theory Prob. Appl. 2, 256-266. 